OAK RIDGE

NATIONAL LABORATORY

\title{
TNG-GENOA USER'S MANUAL
}

F. B. Guimaraes and C. Y. Fu

\author{
October 2000
}

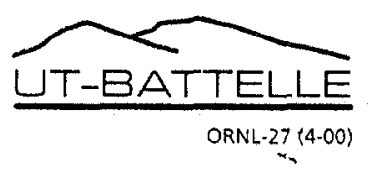


Computational Physics and Engineering Division

\title{
TNG-GENOA User's Manual
}

\author{
F. B. Guimaraes and C. Y. Fu
}

October 2000

\author{
Prepared by the \\ OAK RIDGE NATIONAL LABORATORY \\ managed by \\ UT-BATTELLE, LLC \\ for the \\ U.S. DEPARTMENT OF ENERGY \\ under contract DE-AC05-00OR22725
}




\section{Contents}

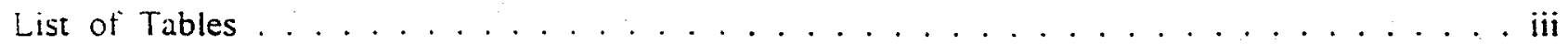

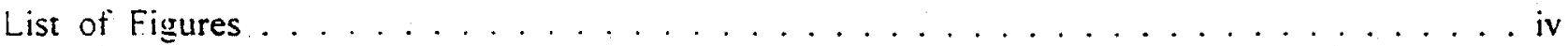

ABSTRACT . . . . . . . . . . . . . . . . . . . . v

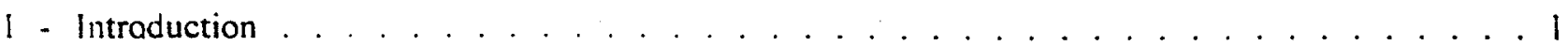

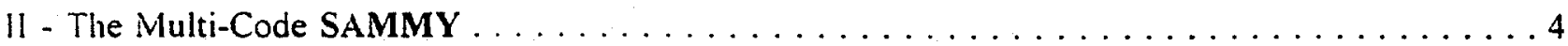

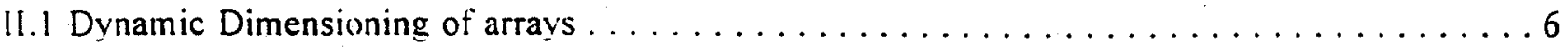

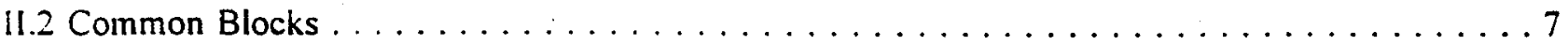

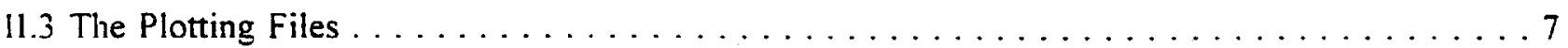

II.4 Sequence of Calculations for GENOA and TNG as part of SAMMY $\ldots \ldots \ldots \ldots \ldots \ldots \ldots$

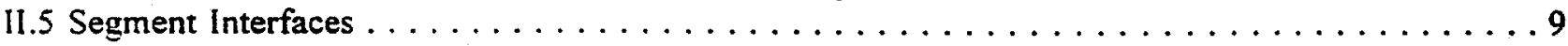

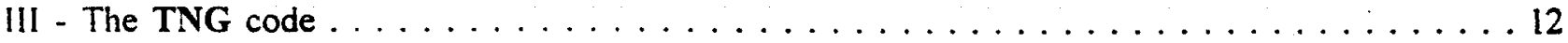

III.1 A consistent description of the Intermediate Nuclear System $\ldots \ldots \ldots \ldots \ldots \ldots \ldots \ldots \ldots \ldots \ldots 12$

III.1.I The Steady-State Component . . . . . . . . . . . . . . . . . . . . . . . . . 14

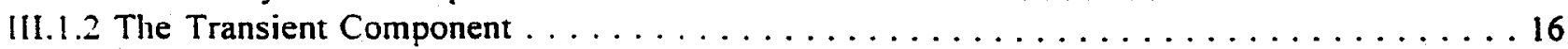

III.1.3 The Particle Emission Spectra in Nuciear Reactions . . . . . . . . . . . . . . . . . . 17

III.2 Spin Population of SP-States in the Pre-Equilibrium Stage $\ldots \ldots \ldots \ldots \ldots \ldots \ldots \ldots \ldots \ldots \ldots$

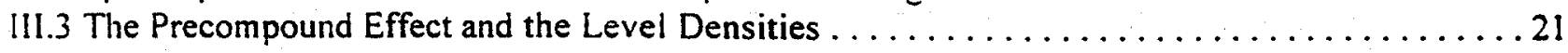

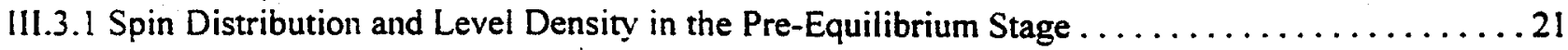

III.3.2 Level Density for the Compound Nucleus . . . . . . . . . . . . . . . . . . . . 23

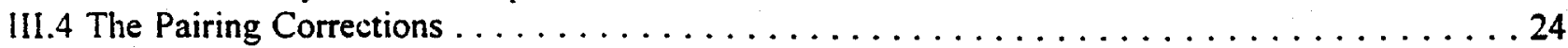

III.4.I The Particle-Hole State Density in One-Fermion Model . . . . . . . . . . . . . 26

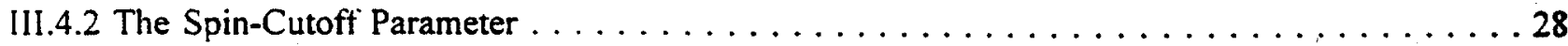

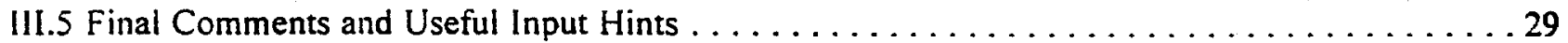

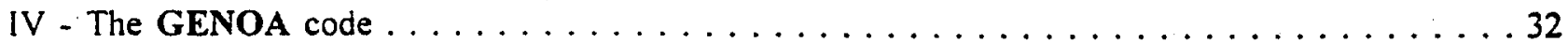

IV.1 Description of the Optical Potential in the GENOA code $\ldots \ldots \ldots \ldots \ldots \ldots \ldots \ldots \ldots \ldots . \ldots \ldots$

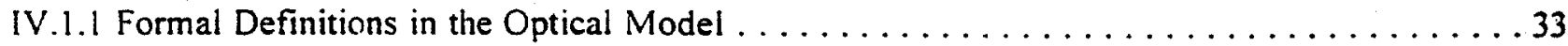

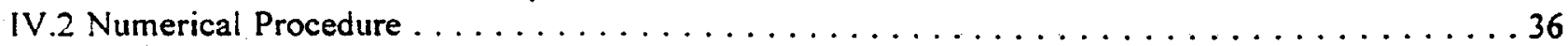

IV.2.1 Numerical Integration of the Schroedinger Equation $\ldots \ldots \ldots \ldots \ldots \ldots \ldots \ldots \ldots \ldots \ldots$

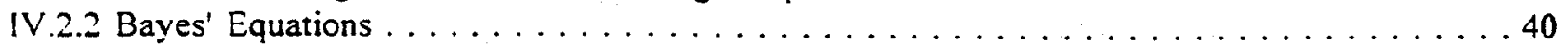

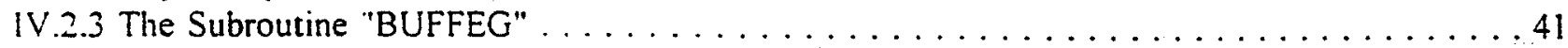

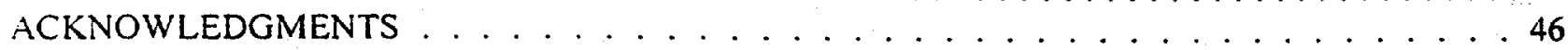

References .. . . . . . . . . . . . . . . . . . . . . . . . . 47

Appendix A - Description of TNG Input (unit-60 and unit-63) $\ldots \ldots \ldots \ldots \ldots \ldots \ldots$

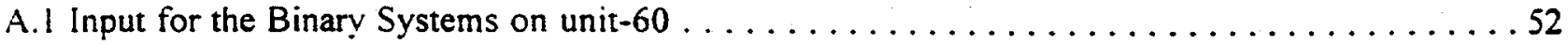

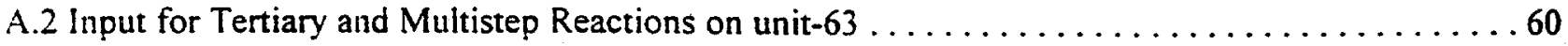

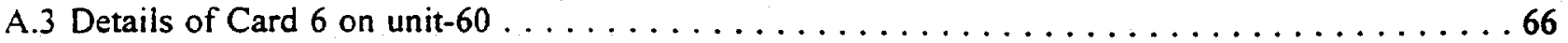

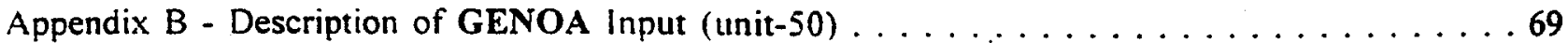

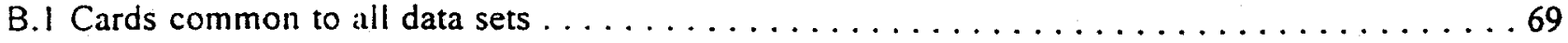

B.2 Specific definitions for each experimental data set $\ldots \ldots \ldots \ldots \ldots \ldots \ldots \ldots \ldots \ldots \ldots \ldots$

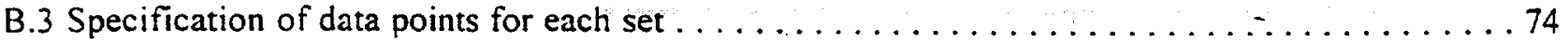

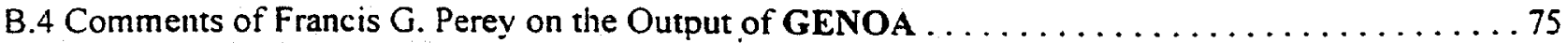

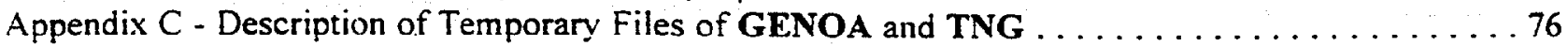


. 


\section{List of Tables}

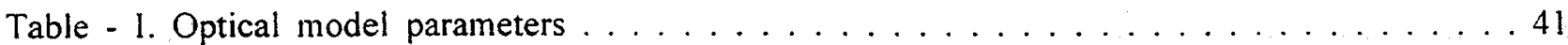

Table - Il. Optical Model Symbols x Variables Used in "BUFFEG" . . . . . . . . . . . 43

Table - III. Number of Central Paramters . . . . . . . . . . . . . . . . ......... . 44

Table - IV. "BUFFEG" Parameter and Relation to Central Parameters . . . . . . . . . . 45 


\section{List of Figures}

Figure 1. Spin populations in $63 \mathrm{Cu}$ following 14.6-MeV $\left(n, n^{\prime}\right)$ reaction for two outgoing neutron energies. $E=4: 5 \mathrm{MeV}$ and $E=8: 5 \mathrm{MeV}$. The solid curves are based on the model implemented in TNG, which includes precompound effects. The dashed curves are based on the Hauser- Feshbach method.... 


\begin{abstract}
The aim of this work is to describe the basic aspects of the codes TNG and GENOA. These codes have been developed and used at ORNL in the last decades for the analysis and evaluation of neutron induced nuclear data. in the energy region of the unresolved resonances range and high energies range. These evaluations have been performed in support of various projects and were included in the ENDF/B library. The implementation of these codes into the code SAMMY has been performed, as part of a program of the creation of a general Nuclear Data evaluation tool for the analysis of reactions in a broad energy range, from few eV up to about $150 \mathrm{MeV}$.
\end{abstract}




.




\section{I - INTRODUCTION}

This paper is intended to be a general purpose manual which describes not only the basic theoretical formalism used in the program definitions of the codes GENOA [] and TNG [4,5], but also details of their program structures and the parameters used in their input files. In addition, we describe the interconnections between GENOA and TNG as parts of the computer code SAMMY.

These codes perform Optical Model calculations (GENOA) and the statistical description of preequilibrium (P-E) and Compound Nucleus (CN) decay (TNG), for the analysis of the unresolved-resonance spectrum of nuclear reactions and high energy interaction.

Generally speaking, the cross-section evaluations of the unresolved and high energy regions could be performed in two stages. The first stage would consist of the generation of the transmission coefficients for the various outgoing particles exciting energy levels, using an Optical Model code. Then, the activation cross-sections for each reaction would be calculated using a statistical model for the pre-equilibrium and equilibrium emissions.

Therefore, GENOA and TNG have complementary roles in nuclear data evaluation of the unresolved and high energy regions.

The pre-equilibrium processes take place before the Compound Nucleus is formed and, therefore, the reaction time is much shorter than in the compound reactions. The corresponding reaction chain can be treated statistically as in the inulti-step direct formalism (MSD) developed by Feshbach, Kerman and Koonin (FKK) [1]].

The processes in nuclear reactions following MSD can be thought as developing along a chain of states of the whole nuclear system of increasing quantum mechanical complexity; due to the increasing number of empty single-nucleon quantum states available for internal transitions below the Fermi level. This theoretical picture is called the multi-step compound (MSC) chain of states. For medium and large nuclei the dynamics of such complex system can also be treated statistically by the formalism of Feshbach-Kerman-Koonin [1]]. These processes can be approximated by the Hauser-Feshbach formalism [2].

The TNG [4,5] code combines the MSD and MSC formalisms, using an extension of the HauserFeschbach theory. This extended model can be used for the analysis of nuclear reactions induced by neutrons in the unresolved-resonance region, with energies up to some few tens of $\mathrm{MeV}$. The parameters of the various reaction channels are calculated using the Optical Potential Model with spin-orbit coupling. The Optical Model (OM) [3,6] applies to processes in which the detailed description of the structures of the incident and 
target particles is not feasible or not necessary. This assumption is usually well suited for the analysis of cross-sections in the unresolved-resonance region, mainly in the region of the continuum. In this region, the individual resonances strongly overlap, which causes the compound cross-section, $\sigma_{C}$, and average reaction cross-section, $\left\langle\sigma_{R}\right\rangle$, to coincide. Therefore, for high energies the compound elastic cross-section, $\sigma_{C E}$, becomes negligible.

The microscopic foundation of the Optical Model is the approximate freedom with which the nucleons move inside a nucleus, which has been evidenced by the great success of the Shell Model for the nuclear ground state [3]. Therefore, it becomes natural to describe the movement of particles inside an excited nucleus as the superposition of motion under the action of a central potential plus an absorption term, both in an average way.

Structurally, the Model consists of a simple quantum-mechanical description of the nuclear reaction in terms of the scattering of the incident particle by a central local potential. This potential is assumed to be complex to account for the non-elastic reactions, including the fission channel [7].

The GENOA code []ㅛ performs a searching procedure to determine the parameters of a phenomenological global Optical Potential, i.e., a potential capable of fitting nuclear data for a wide range of target nuclei mass numbers and reaction energies, with a single set of parameters. Some parameters may depend on the reaction energy or on the mass of the target nucleus.

There is a simple numeric connection between these two codes. On one hand, GENOA is used to fit the selected sets of elastic angular distribution data as well as the total cross sections. This calculation produces an Optical Potential which can be directly used by TNG as an input option for the reaction potential. Complementary, TNG calculates compound elastic angular distributions as a function of the incident particle energy. Then, the resulting Legendre coefficients, for each angular distribution, can be used by GENOA for the production of more precise OM-parameters.

This connection has been exploited whenever the two codes were simultaneously used for Nuclear Data evaluation [ $[\underline{9,10}]$ and, also, for the implementation of TNG and GENOA into SAMMY. [11]

Some aspects of this implementation are described in Sec.II. In Sec.III a general description of theoretical aspects of the pre-equilibrium and Hauser-Feshbach models used in TNG is given. A short definition of the input files parameters is given in Sec.III.5.

In Sec.IV we present a general description of GENOA code and the numerical fitting process based on Bayes' equation for conditional probability evaluation, which is the same used in other segments of the multi-code SAMMY. 
A detailed description of input files for TNG can be found in Appendix A, while Appendix B describes the input data for GENOA. At last, in the Appendix C a detailed description is given of the temporary - and output files generated by a run of the connected code TNG and GENOA (as parts of SAMMY). 


\section{II - THE MULTI-CODE SAMMY}

Until recently, the codes SAMMY, TNG and GENOA have been independently used to nuclear data evaluations $[9,10,12,13]$. In particular, SAMMY has been used in the analysis of the nuclear data in the resolved-resonance region [14,15].

The combinations of computer codes, like TNG and GENOA, into SAMMY gives a unique capability for data evaluation in the energy range from few $\mathrm{eV}$ up to about $40 \mathrm{MeV}$. To the.best of our knowledge there is no such capability available elsewhere.

SAMMY has a large programming structure, consisting of various segments [11] dynamically connected to an initialization "master" program, which are sequentially and independently executed. Technically, this is performed through the definition of large "dummy" matrices of variable dimensions, for more efficient memory allocation, and the definition of temporary data storage files. In addition, a linking Clanguage program, called "execv.c", is used to perform the ordered and sequential execution of the diverse segments.

The structure of SAMMY allows the implementation of successive new parts, which may be important for nuclear data analysis, with great independence with respect to the other parts of the code. In this way, the change or update of a segment does not interfere with the remaining segments that may have already been updated or that need not be updated.

For example, the segment "saminp" reads and processes SAMMY's input file, usually defined with the extension ".inp", which have the basic information for running SAMMY. The segment "sampar" reads and processes the parameter file, usually with extension ".par", which contains the resonance parameters. The segment "samxct" calculates the cross-sections, which may be Doppler-broadened in "sarnfgm" (free gas model) and/or resolution-broadened in "samrsl".

The actual fitting is performed in the segment "sammpw". Other possible segments for fitting are "samnpv" and "samipq", but in the future only "sammpw" shall be used. Two arrays are required as input for "sarnmpw", called respectively " $Y$ " and " $W$ ", and are passed between "sammpw" and the other segments of SAMMY through the storage of the FORTRAN arrays "xxoxxx" and "wwwwww", respectively, in adequate temporary files.(see Appendix C)

More details on the numerical fitting procedure can be found at the end of this Manual, in Sec.IV.2 See also Sec.II.5, for details on the segmentation structure of TNG-GENOA within SAMMY. A comprehensive description of the temporary files can be found in Appendix C. 
For the implementation of TNG and GENOA into SAMMY, the division of their subprograms into segments, according to SAMMY's structure, has split GENOA into 3 parts and TNG into 2 parts.

For TNG the parts and the corresponding independent programs are the following,

$\begin{array}{ll}\text { operation } & \frac{\text { segment }}{\text { initialization }} \\ \text { numeric calculations } & \text { "samtng" }\end{array}$

and for GENOA the various parts are,

$\begin{array}{ll}\text { operation } & \text { segment } \\ \text { initialization } & \text { "samgen" } \\ \text { numeric fitting calculations } & \text { "sammpw" } \\ \text { optical potential calculations } & \text { "samgit" }\end{array}$

In general, the executable files of SAMMY are named in a systematic fashion. The generic executable "samX" is generated with the source files stored in the corresponding subdirectory "/X/". In the particular case of the executables "samtn1" and "samtng", both are generated with the source files stored in the subdirectory "/tng/". The executables associated with GENOA, "samgen" and "samgit", are compiled from the source files located in the directory "/gen/". All the executable files are located in one subdirectory, usually called "/exe/", and are sequentially run, using the program "execv", beginning by the "master" executable, called "sammy".

The sequential run of segments is performed with the help of an specific subroutine, named "run", located in the source file "/end/msamxx.f", which dynamically defines the paths of the various independent programs and calls "execv" with each newly generated path.

The files which contain both subprograms, "execv" and "run", are located in the same subdirectory, "/end/". Usually, an independent part of SAMMY is defined ("samend"), using the files of this subdirectory, which performs the final definitions for adequate memory allocation at the end of each iterative procedure. To run GENOA and TNG within SAMMY only a few subroutines from the "/end/" directory are necessary and the independent program "samend" must not necessarily be used. 


\section{II.1 DYNAMIC DIMENSIONING OF ARRAYS}

In almost every segment of SAMMY, the arrays are not defined explicitly but are pulled out of the "container" array in the block "common/expand/a(n)" [11] $]$, where " $n$ " is usually a large number. The dynamic dimensioning feature of SAMMY was extènded to TNG and GENOA.

For example, in the segment "samgen" two subroutines of GENOA are used in the dynamic dimensioning procedure. The subroutine "gnpass" is used to provide a first pass through the input data file to get the dimensions, which are effectively assigned in the subroutine "gnsetd ". Both subroutines are located in the file"/gen/genoa.f".

To clarify this point we consider an specific example. In GENOA an array named "xst", which has dimension equal to the number ("ngnpar") of parameters, is used in the GENOA's search procedure to define the Optical Potential. In this case, the parameter "ngnpar" is read in subroutine "gnpass" and subroutine "gnsetd" contains the FORTRAN command

$$
\text { ignxst }=\text { idimen(ngnpar) }
$$

Then, the resulting parameter "ignxst" is used to define a subset of locations in the array "a(n)", beginning at the position "ignxst" with dimension "ngnpar".

In the above exarnple, the function "idimen" keeps track of the last-used location in "a(*)" and assigns the next spot as the beginning of array "xst".

After the definition of the dimension, the FORTRAN call-command to subroutine "iogeno", which uses the array "xst", has the form,

$$
\begin{aligned}
& \text { CALI IOGENO( } \\
& >a(\text { ignxst), a(igxcen), a(ignipa),a(igntsp), a(ignkrc), a (ignarr), } \\
& >\text { a(ignkar), a(igatkp), a(ignang), a(ignexp), a (ignwgt), a(igndel), } \\
& >\mathrm{a}(\text { ignxco), a(igarra), a(igarre),a(igarrw), a(ignsns)) }
\end{aligned}
$$

where the other indices, "igxcen", "ignipa", etc., corresponding to the arrays "xcen", "ipa", etc., have similar definitions, using "gnpass" and "gnsetd". Finally, subroutine "iogeno" itself starts out as, 


\section{SUBROUTINE IOGENO(xst,xcen,xipa,tsp,xkrc,arr,xkar,atkp, >angexp,fexp,weight,delsig,xcomp,arra,arre,arrw,compe) \\ $\operatorname{dimension} x s t\left({ }^{*}\right), \mathrm{xcen}\left({ }^{*}\right), \mathrm{ipa}\left({ }^{*}\right), \operatorname{tsp}(*), \ldots$}

with variable dimensions assigned to the various arrays of the subroutine's argument.

\section{II.2 COMMON BLOCKS}

Common blocks which are used repeatedly in SAMMY are kept in separate files located in the subdirectory "/blk/. For instance, the file "/blk/TRANSF", which is used mainly in the definition of temporary files, must be included in the source program files which use it ("/ger/genoa.f", "/gen/wrt69.f", "/git/sgenoa.f" and "/mpw/rnmpw.f"), using the FORTRAN command,

$$
\text { include 'TRANSF' . }
$$

The corresponding compile command must also include the path name to access these files. For example, to compile "genoa.f" in the "no hang up"-mode (UNIX "nohup"-command) the script line is,

$$
\text { nohup } \mathrm{f77}-\mathrm{c}-\mathrm{I} . . / \text { blk genoa.f } \gg \text { nvnv }
$$

where "nvnv" is the output file which appends the FORTRAN messages for all compilations in the given subdirectory.

\section{II.3 THE PLOTTING FILES}

In SAMMY, the files to be used for plotting purposes are the ODF-files (ORELA Data Format-files), with extension ".odf". These files are written in binary mode and can be used for direct plotting with a Tektronix terminal. 
Usually SAMMY gives only one plotting output file, called "SAMMY.ODF", but in TNGGENOA segment of SAMMY the plotting information is directly recorded in various ODF-files, which have names defined sequentially for each independent reaction. In this way, the theoretical results are stored into the files "stgtyy.odf", where "yy" is the ordering number of the specific reaction for which the cross-section has been calculated. It stores the "best" fitted cross-section angular dependence, as calculated by GENOA, for the given reaction. The corresponding experimental cross-section angular dependence is recorded in the "stgxyy.odf" files.

These files are written in the unit-70 in subroutine "plogen", which is located in the file"/gen/wrt69.f". Subroutine "plogen" is a modified version of "plott1", in the file "/fff/mfff2.f". The subroutine "plott1" is used to generate plotting files in the segment "samfff" of SAMMY, which corresponds to the implementation of code FITACS into SAMMY. [11]

The subroutine "plogen" is called in the subroutines "iogeno", in the source file "/gen/iogeno.f" of the segment "samgen" (for experimental data), and "fitleg", in the file "/git/fitleg.f" of the segment "samgit" (for evaluated data).

\section{II.4 SEQUENCE OF CALCULATIONS FOR GENOA AND TNG AS PART OF SAMMY}

The general sequence of calculations performed by the GENOA-TNG block is as follows. Initially, the main subprogram of GENOA is called by SAMMY

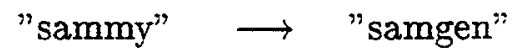

to initiate a series of iterations

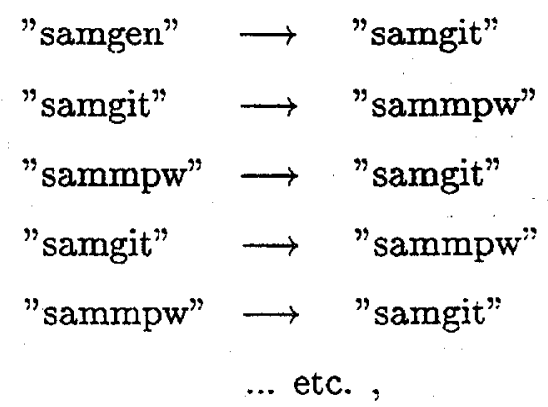

to determine the "best" Optical Potential which will describe a given set of reactions.

After convergence, GENOA calls the main subprogram of TNG, 


\section{"samgit" $\quad \longrightarrow$ "samtn1"}

which reads the resulting optical potential parameters and calls "samtng",

"samtnl" $\longrightarrow$ "samtng"

to perform statistical pre-equilibrium and equilibrium calculations and generate the Legendre coefficients.

At last, the resulting Legendre coefficients are used to redefine the input of GENOA and "samgen" is called again by $\mathbf{T N G}$,

$$
\text { "samtng" } \longrightarrow \text { "samgen" }
$$

The whole sequence repeats until a given precision is achieved or a given number of iterations is performed.

The basic quantity used to measure the precision is the " $\chi^{2 "}$ parameter defined by,

$$
\chi^{2}=\frac{1}{N} \sum_{i=1}^{N}\left(\frac{\sigma_{t h}\left(\theta_{i}\right)-\sigma_{e x p}\left(\theta_{i}\right)}{\Delta \sigma_{e x p}}\right)^{2},
$$

where, $\sigma_{\text {exp }}$ and $\sigma_{\exp }$ are, respectively, the calculated and experimental values of the cross-section at the scattering angle $\theta_{\text {exp }}$ and $\left(\Delta \sigma_{e x p}\right)^{-2}$ is the statistical weight of the point $\sigma_{\text {exp }}$. In GENOA $\Delta \sigma_{\text {exp }}$ is taken as the experimental error.

This expression for $\chi^{2}$ assumes that the errors are not statistically correlated, but this may not be true (see Ref. [17] for a detailed analysis of this fitting criterium).

Another useful convergence parameter is

$$
F_{\chi}=\left(\frac{\chi_{p r e}^{2}-\chi_{b e f}^{2}}{\chi_{p r e}^{2}}\right),
$$

which is more useful when a large number of data for different reactions has to be analyzed simultaneously. Here, $\chi_{\text {pre }}$ and $\chi_{\text {bef }}$ are, respectively, the present and the former values of the $\chi$ parameter.

\section{II.5 SEGMENT INTERFACES}

Beyond the definition of the adequate program block structure, it is necessary to implement the numerical and physical "interfaces" among the various connected blocks of the code.

The "numerical interface" is simpler to construct and consists of the careful definition of the I/O parameters to be exported between the sequentially connected blocks. This is done with the help of temporary files which store the data of the container array "a(n)", referred in Sec.II.1, as well as some specific arrays of the "MPW" procedure, like "xcen", "xoxoxx", "wwwwww", etc. 
In particular, the common block "TRANSF" has been specifically defined to help in the data transfer between segments of GENOA within SAMMY, in connection with the source file "/gen/wrt69.f". This data transfer generates the ternporary files "STG72.DAT", "SAM11.DAT" and "SAM69.DAT" and permits the use of the "MPW" segment of SAMMY, "sammpw" referred above, to perform the numerical fitting procedure in GENOA.

Another source file, "/gen/wrt150.f", uses the information in the temporary files "STGA56.DAT" and "STGA71.DAT" to generate an updated input file of GENOA, "fort.50", after each iterative evaluation of the OM parameters. The updated file can be used to begin a new set of iterations if the total number of runs (variable "icrun") is bigger than one. (see Card 1 in Appendix B)

The "physical interface" is more complex. having in sight that the appropriate formalisms to analyze nuclear reaction data in the various energy regions are, in general, quite different from one another. This difference results in part from the different precision with which the spectra in the various energy regions are measured, but also from the different physical phenomena which appear in the different regions.

For the definition of the "physical interface", the parameters obtained in a given block of the code or a given step of the calculations must be used to automatically generate another set of parameters, adequate for the analysis of the spectrum in another block or step. In addition, for a given energy region the input parameters must be varied whenever possible and the internal parameters of the corresponding phenomenological model must be adjusted to accomplish the best fit of the experimental data in that region.

For exarnple, in GENOA not only the parameters of the OM potential can be varied, but also the auxiliary ones associated with the determination of the compound elastic cross-section. The automatic variation of these two sets of parameters gives, in general, a good fit to experimental data for one reaction or a set of reactions with energies close to each other.

On the other hand, when a greater number of reactions in a larger range of incident energies is considered simultaneously, the cross-section evaluation cannot be performed in a straightforward way. Instead, it is necessary to analyze initially each individual reaction and in a second step perform the evaluation of the whole set of reactions.

Due to these inultiple intermediate steps, the total fitting in the more complicate cases cannot, in general, be performed automatically, because a parameter which gives a good description of one reaction usually must be modified to describe a larger set of reactions, with various levels of experimental precision and various reaction energies. 
In summary, the "physical interface" procedure is as automated as possible and consists of a physical analysis of the numerical results between blocks of the code or calculation steps, which may include the execution of numerical averages when going toward regions of higher reaction energies. In the latter case, the evaluated spectra must match smoothly in the boundaries between any two of these regions.

In the GENOA-TNG segment of SAMMY this interface is not yet fully implemented because some steps are not yet automatic. Therefore, the evaluation of a complicate set of reaction data may need multiple redefinition of the codes' input, in a sequence of independent jobs. 


\section{III - THE TNG CODE}

The TNG code is based on a formalism which consistently unifies the statistical models of HauserFeshbach (H-F) and for pre-equilibrium (P-E) processes [4]. It uses a modified exciton model [18] for the description of precompound emission, which automatically reduces to the usual evaporation formula after the equilibrium has been reached.

In the following sections we give a general description of this model and its consequences; more details can be found in Refs. $[\underline{4,5,19,20]}$

\section{III.1 A CONSISTENT DESCRIPTION OF THE INTERMEDIATE NUCLEAR SYSTEM}

In the exciton model an excited many-body system is characterized by a simple quantum-mechanical description, in terms of the occupation numbers of the single-particle (sp) states. This is inspired on the Shell Model characterization of the non-excited system as a set of filled sp-states of increasing energies, up to a maximum called the system's Fermi level.

In the excited systern, the filled sp-states above the Fermi level are defined as excitons of particle-type, while the empty sp-states below the Fermi-level are defined as excitons of hole-type. The total number of particles in this system is usually designated by " $p$ ", while the total number of holes is designated by " $h$ ". Thus, the exciton number is defined by the sum "p+h", usually designated by " $n$ ".

Consequently, to each exciton number " $n$ " is associated a whole class of states of the excited manybody system, corresponding to all possible ways by which the " $p$ " particles and " $h$ " holes can be distributed within the available levels of the system, for a given excitation energy. Therefore, the probability associated with the description of a given sp-state becomes a distribution over the number of excitons " $n$ " and the many-body system excitation energy " $E$ ".

At the end of the pre-equilibrium chain, the equilibrated excited system is identified with the Compound Nucleus. The evaluation of the decay spectra is characterized by integrations of the equation of state along the intervals " $[0, T]$ ", for the pre-equilibrium emission, and "[T, $\infty$ ", for the $C N$ emission, where $T$ is the time interval necessary for the equilibrating system to reach the Compound Nucleus stage. 
Both the $C N$-system and the P-E nuclear states define a quasi-equilibriun $(Q E)$ nuclear many-body system, in the sense that these systerns are not stable but decay after a finite tirne interval. The general equilibration formalism is, therefore, defined in terms of the $Q E$-states and their possible transitions.

Let " $P_{\nu}(n)$ " be the occupation probability distribution of sp-states filled with particles of type " $\nu$ ", belonging to the class of $Q E$-states with exciton number " $n$ ". Then, its tirne rate of charige is defined by the following "master equation", [4,21,22]

$$
\begin{aligned}
\frac{d P_{\nu}(n)}{d t} & =\left[P_{\nu}(n-2)\left(\frac{p-1}{p}\right)+\frac{f_{\nu}(p)}{p} P(n-2)\right] \lambda^{+}(n-2)+ \\
& P_{\nu}(n+2) \lambda^{-}(n+2)-P_{\nu}(n)\left[\lambda^{+}(n)+\lambda^{-}(n)+\int_{0}^{\left(E-B_{\nu}\right)} X_{\nu}(n, \epsilon) d \epsilon\right]
\end{aligned}
$$

where " $E$ " is the kinetic energy of the emitted $\nu$-particle, " $B_{\nu}$ " is its binding energy before emission and, therefore, " $E-B_{\nu}$ " is the maximum kinetic energy available for the emitted $\nu$-type particle.

The functions $\lambda^{+}(m)$ and $\lambda^{-}(m)$ are the "internal" rates of transition from QE-states with initial exciton number " $m$ " to states with final exciton number " $m+2$ " and " $m-2$ ", respectively. These rates describe a steady equilibrium state of the excited nuclear system and do not include the emission process.

The "internal" characteristic of $\lambda^{+}(m)$ and $\lambda^{-}(m)$ means that, if one does not consider the decay of the $Q E$-system, the various multi-exciton states are different dynamical manifestations of the same excited nuclear system in the pre-equilibrium chain, defined by the intra-nuclear interactions, the conservation of energy and momentum and Pauli's exclusion principle. Opposed to this are the absorption and emission processes which define a break in the P-E chain, generating other P-E systenns which will evolve along their own chains.

In Eq.(3.1), the departure from equilibrium is determined by the integral term, which describes the rate of decrease of the occupation probability of sp-states due to emission of $\nu$-type particles at the time "t". (see Sec.III.1.2)

The function " $f_{\nu}(n)$ " is the probability of finding particles of type " $\nu$ " in a newly excited n-excitons nuclear state, resulting from the excitation of one $\nu$-particle (and one $\nu$-hole) on a nuclear state with $\left(n-2 n_{\nu}\right)$-excitons, where $n_{\nu}$ is the total number of excitons associated with the creation of a "particle" of type " $\nu "$.

Therefore, the function " $f_{\nu}(n)$ " describes a perturbed situation which is complementary to the steadystate described by " $P_{\nu}(n)$ ", " $\lambda^{+} "$ and " $\lambda^{-}$", but this perturbation is assumed to be weak enough so that the nuclear steady-state is not altered. 
To simplify the general description of the model and avoid for awhile the consideration of clusters of nucleons and "condensation" processes associated with the emission of complex particles, we assume in (․1.) that " $n_{\nu}$ " is one, corresponding to protons or neutrons.

The function " $f_{\nu}(n)$ " is a distribution over the types of particle of the model, satisfying the normalization condition

$$
\sum_{\nu} f_{\nu}(p)=1
$$

for a nuclear system with a given number of excited particles " $p "$.

The consistency of the above description of the whole equilibration process lies on the specific definition of a master equation for each component, $P_{\nu}(n)$, of the emission spectrum. This avoids the use of "ad hoc" probability parameters to describe the relative presence of " $\nu$ "-type particles among the total "p" excited ones, for nuclear states with given " $n$ " and " $E$ ", at a given instant " $t$ " of the dynamical evolution of the $Q E$-system. [22]

This formulation is equivalent to the definition of a time dependent probability function, for finding a " $\nu$ "-type particle in $Q E$-states with $n$ excitons, given by

$$
R_{\nu}(p, t)=\frac{P_{\nu}(n, t)}{P(n, t)}
$$

which satisfies,

$$
\sum_{\nu} R_{\nu}(p, t)=1
$$

and equation ( $\underline{3.1})$. Therefore, this is the analog of the perturbed function " $f_{\nu}(p)$ ". for the unperturbed steady-state and can be used for the consistent evaluation of cross-sections along the whole equilibration process.

To obtain a nurnerical evaluation of the equilibrium and non-equilibrium terms of equation ( $\underline{3.1}$ ) TNG uses an specific microscopic description for each term, as explained in the following two sections.

\section{III.1.1 The Steady-State Component}

From the time-dependent perturbation theory one can obtain a simple approximate relation for the time rates of transition between single-particle excited states in the $Q E$-system. 
If the residual interactions which produce the transitions are sufficiently weak, the nnany-body equilibrium is approximately not altered by the single-particle transitions. In this case, the transition rates are proportional to the square of the matrix elements of the residual interaction potential, calculated between the initial and final sp-states, and to the total nurnber of final sp-states of the Iruclear system available for that transition, at the equilibrium.

This basic result, also known as the "Fermi's Golden Rule", can be formulated as [21,23]

$$
\lambda=\frac{2 \pi}{\hbar} \sum_{f}\left|\left\langle i\left|V_{\text {residual }}\right| f\right\rangle\right|^{2} \delta\left(\epsilon_{f}-\epsilon_{i}\right)
$$

where $\lambda$ is the transition rate, "|iो" and " $|f\rangle "$ are, respectively, the initial and final sp-states of the $Q E$ system, with energies $\epsilon_{i}$ and $\epsilon_{f}$, and the sum over final states include all possible transitions compatible with the residual interaction, the conservation laws, and Pauli's exclusion principle.

Assuming that the residual interaction does not depend strongly on the final sp-states one can factor its mean value, $\left|\left\langle i\left|V_{\text {residual }}\right| f\right\rangle\right|^{2}$, and rewrite the above expression schematically as, [16,22]

$$
\lambda^{\Delta n}=\frac{2 \pi}{\hbar} M_{\Delta n}^{2} Y
$$

where $M_{\Delta n}^{2}$ is the average square matrix-element for two-body interactions between an specific initial state and any possible final state, cornpatible with the given exciton number. The residual interaction is assumed to be of two-body type, therefore, the variation in exciton number between initial and final sp-states is $\Delta n= \pm 2,0$. Strictly speaking, $M_{\Delta n}^{2}$ is a function of $\Delta n$, but this dependence will be neglected here.

The parameter $Y$ is the accessible phase space for the transition, that is, the total number of available final states, for possible transition for a given excitation energy interval $[E, E+d E]$, including spin degeneracy. Assuming an equidistant level spacing model for the sp-energy spectrum, with distance between levels " $g$ ", $Y$ is given by the density of final sp-states of an n-exciton nuclear system,

$$
Y(n, E)=\omega(n, E)=\frac{g(g E)^{n+1}}{p ! h !(n-1) !}
$$

Equations (‥5) and ( $\underline{3.6})$ permit the evaluation of $\lambda^{+}(m)$ and $\lambda^{-}(m)$ in a phenomenological way. The explicit expressions which take into account Pauli's exclusion principle were originally obtained by Williams $\underline{33,4}]$

$$
\begin{aligned}
& \lambda^{-}(p, h, E)=\frac{2 \pi}{\hbar} M^{2}\left(\frac{g}{2}\right) p h(p+h-2) \\
& \lambda^{+}(p, h, E)=\frac{2 \pi}{\hbar} M^{2}\left(\frac{g}{2}\right) \frac{\left(g E-C_{p+1, h+1}\right)^{2}}{(p+h+1)}
\end{aligned}
$$




$$
\lambda^{0}(p, h, E)=\frac{2 \pi}{\hbar} M^{2}\left(\frac{g}{2}\right) \frac{\left(g E-C_{p, h}\right)}{(p+h)}\left(p^{2}+h^{2}+4 p h-p-h\right)
$$

where $\lambda^{0}$ is the pararneter for transitions between $Q E$-states with the same number of excitons and $C_{p, h}$ is the Pauli's principle correction factor given by

$$
C_{p, h}=\frac{1}{4}\left(p^{2}+h^{2}+p-3 h\right)
$$

\section{III.1.2 The Transient Component}

In equation ( $\underline{3.1})$ the function " $X_{\nu}$ " measures the rate, per unit time, of emission of particles of type $\nu$ from $Q E$-states. It is a distribution over the exciton-number of the $Q E$-system, " $n$ ", and the energy of the emitted particle, " $\epsilon_{\nu}$ ". It defines a decrease of the occupation probability for a given exciton-number and can be estimated using the principle of "detailed balance" 24,25$]$

$$
W_{Q R}=\frac{N_{R}}{N_{Q}} W_{R Q}^{*}
$$

where $W_{Q R}$ is the probability per unit time that the nuclear system in a given state belonging to the set "Q" (quasi-equilibrium class of states before decay) will undergo a transition to any of the states belonging to the set " $R$ " (residual nucleus). The parameter $W_{R Q}^{*}$ corresponds to the time-reversed process, for the probability per unit time that the nuclear system in a state in $R$ (time-reversed) will undergo a transition into any of the $Q E$-states belonging to $\mathrm{Q}$ (time-reversed).

$N_{Q}$ and $N_{R}$ are the total number of sp-states in the sets $Q$ and $R$, respectively, and are given by the product of the corresponding state-densities times the energy interval,

$$
N_{Q}=\omega(p, h, E) \mathrm{d} E
$$

and

$$
N_{R}=\omega(p-1, h, E) \mathrm{d} E \omega_{\nu}\left(\epsilon_{\nu}\right) \mathrm{d} \epsilon_{\nu}
$$

where $\omega(n, E)$ is the density of sp-states in the $Q E$-system, with total excitation energy $E$ and exciton number $n$. The excitation energy of the residual nucleus " $U$ " is related to the energy of the $Q E$-system before emission, " $E$ ", by the relation

$$
U=E-B_{\nu}-\epsilon_{\nu}
$$


where " $B_{\nu}$ " is the binding energy of the ernitted particle.

The term " $\omega_{\nu}\left(\epsilon_{\nu}\right) \mathrm{d} \epsilon_{\nu}$ " is the total number of free $\nu$-particle states in the final channel.

The time-reversed transition probability $W_{R Q}^{*}$ can be written in terms of the $\nu$-particle capture crosssection, $\sigma_{\nu}$, and the energy of the emitted particle in the direct process, $\epsilon_{\nu},[24,25]$

$$
W_{R Q}^{*}=\frac{v_{\nu} \sigma_{\nu}\left(p-1, h, \epsilon_{\nu}, U\right)}{V}
$$

with the velocity in the CM-system given by,

$$
v_{\nu}=\left(2 \epsilon_{\nu} / \mu_{\nu}\right)^{1 / 2}
$$

and $\mathrm{V}$ is the volume to which the free particle " $\nu$ " is confined. The density of $\nu$-particle states can then be evaluated as the quantum-mechanical solution for the sp-states density for a confined free particle

$$
\omega_{\nu}\left(\epsilon_{\nu}\right) \mathrm{d} \epsilon_{\nu}=\frac{V}{4 \pi^{2} \hbar^{3}}\left(2 s_{\nu}+1\right)\left(2 \mu_{\nu}\right)^{3 / 2} \epsilon_{\nu}^{1 / 2} \mathrm{~d} \epsilon_{\nu}
$$

where " $\left(2 s_{\nu}+1\right) "$ is the spin degeneracy factor and $\mu_{\nu}$ is the reduced mass of the system composed of the $\nu$-particle and the residual nucleus,

$$
\mu_{\nu}=\frac{m_{\nu} m_{\text {residual }}}{m_{\nu}+m_{\text {residual }}}
$$

Then, " $X_{\nu}$ ", the rate per unit time of emission of $\nu$-particles, is given by

$$
X_{\nu}\left(n, \epsilon_{\nu}\right)=W_{Q R}=W_{\nu}\left(n, \epsilon_{\nu}\right) \mathrm{d} \epsilon_{\nu}=\left(\frac{2 s_{\nu}+1}{\pi^{2} \hbar^{3}}\right) \mu_{\nu} \sigma_{\nu}\left(\epsilon_{\nu}\right) \epsilon_{\nu} d \epsilon_{\nu} \frac{\omega_{\nu}(p-1, U)}{\omega(p, E)}
$$

In this way, for any state of the nuclear system with a given number of excitons $n$ and a given excitation energy $E$, the integral term in Eq.(3.1) gives the total variation in the occupation number of $\nu$-type sp-states, due to particle ernission.

The next step in our formulation is to use these results to evaluate the cross-section for emission of $\nu$-type particles as a function of the reaction energy (spectrum).

\section{III.1.3 The Particle Emission Spectra in Nuclear Reactions}

The emission spectrum for particles of type " $\nu$ " up to the equilibration time "T" is given by the product of the cross-section for the formation of the $C N$-nucleus, $\sigma_{C N}$, times the probability of decay of the $Q E$ nuclear system, during the time interval " $[0, T]$, by emission of a $\nu$-type particle 


$$
\sigma_{\nu}^{p r e}\left(\epsilon_{\nu}\right) d \epsilon_{\nu}=\sigma_{C N} \sum_{p} X_{\nu}\left(n, \epsilon_{\nu}\right) d \epsilon_{\nu} \int_{0}^{T} P(p, h, t) \mathrm{d} t
$$

which gives, using Eq. (‥11);

$$
\begin{gathered}
\sigma_{\nu}^{p r e}\left(\epsilon_{\nu}\right) d \epsilon_{\nu}=\sigma_{C N} \\
\times \sum_{p}\left[\left(\frac{2 s_{\nu}+1}{\pi^{2} \hbar^{3}}\right) \mu_{\nu} \sigma_{\nu}\left(\epsilon_{\nu}\right) \epsilon_{\nu} d \epsilon_{\nu} \frac{\omega_{\nu}(p-1, U)}{\omega(p, E)}\right] \int_{0}^{T} P(p, h, t) \mathrm{d} t
\end{gathered}
$$

Therefore, the total pre-equilibrium plus $C N$ emission spectra can be written as

$$
\begin{aligned}
& \sigma_{\nu}^{\text {total }}\left(\epsilon_{\nu}\right) d \epsilon_{\nu}=\sigma_{C N}\left(\frac{2 s_{\nu}+1}{\pi^{2} \hbar^{3}}\right) \mu_{\nu} \sigma_{\nu}\left(\epsilon_{\nu}\right) \epsilon_{\nu} d \epsilon_{\nu} \\
& \quad \times\left[\sum_{p} \frac{\omega_{\nu}(p-1, U)}{\omega(p, E)} \int_{0}^{T} P_{\nu}(p, t) d t+\sum_{p} \omega_{\nu}(p-1, U) \int_{T}^{\infty} \frac{P_{\nu}(p, t) d t}{\omega(p, E)}\right]
\end{aligned}
$$

In these equations the number of holes is assumed to vary by the same amount as the number of particles, therefore, the sum over the number of particles is in fact a sum over the number of excitons varying in steps of two units.

Another important aspect of the exciton model used in TNG is the conservation of the angular momentum, in a form cornpatible with the Hauser-Feshbach formula, which we describe in the next section.

\section{III.2 SPIN POPULATION OF SP-STATES IN THE PRE-EQUILIBRIUM STAGE}

In the description of the stages of a multi-step precompound process the angular momentum may not be conserved in all steps of the equilibration. The problem shows up in the evaluation of the second and further steps in the multi-step formulation of the precompound spectra.

In multistep H-F calculations containing precompound effects, the pre-equilibrium components in the first step, binary-emission reactions, can be calculated using the expression (3.12), which we repeat here,

$$
\sigma_{\nu}^{p r e}\left(\epsilon_{\nu}\right) d \epsilon_{\nu}=\sigma_{C N} \sum_{p} X_{\nu}\left(n, \epsilon_{\nu}\right) d \epsilon_{\nu} \int_{0}^{T} P_{\nu}(p, t) d t
$$

or its approximations, and the results can then be combined with the compound cross sections calculated with the Hauser-Feshbach method [2]]. 
In the second step of calculation, to determine the tertiary-emission reactions, the spin populations in the intermediate nuclei are required but are not available from the precompound part of the calculation [4]. Then, some arbitrary assumption must be made about the spin populations in the intermediate nuclei for the precompound part, violating the rules for angular-momentum conservation.

To define the spin distribution for intermediate pre-equilibrium states, we take into account the spin direction in the principle of detailed balance and decompose the emission rate, defined by Eq. (3.11), into its spin-dependent components $[24,26]$

$$
X_{\nu}\left(n, J, I, E, \epsilon_{\nu}\right) d \epsilon_{\nu}=\frac{1}{2 \pi \hbar} \sum_{s^{\prime} l^{\prime}} T_{\nu s^{\prime} l^{\prime}}^{J}\left(\epsilon_{\nu}\right) d \epsilon_{\nu} \frac{\rho_{\nu}(p-1, I, U)}{\rho(p, J, E)}
$$

where the diverse parameters are

\begin{tabular}{c} 
parameter \\
\hline$T_{\nu s^{\prime} l^{\prime}}^{J}$ \\
$J$ \\
$I$ \\
$s^{\prime}$ \\
$l^{\prime}$
\end{tabular}

description

Optical-Model trarısmission-coefficient

total Spin of the reacting system

Spin of the residual level

channel Spin of the emitted particle $\nu$

Orbital Angular Momentum of the emitted particle $\nu$

and the spin-dependent level density, $\rho(p, h, J, E)$, is given by its relation to the single-particle state density, $\omega(p, h, E)$, by the expression

$$
\rho(p, h, J, E)=\omega(p, h, E) R(n, J)
$$

where the distribution of spins of the excited system is $[\underline{27}]$

$$
R(n, J)=\frac{(2 J+1)}{2(2 \pi)^{1 / 2} \sigma^{3}(U, n)} \exp \left[-\left(J+\frac{1}{2}\right)^{2} / 2 \sigma^{2}(U, n)\right]
$$

and the spin-cutoff parameter, $\sigma^{2}(U, n)$, is given most generally by the expression of Ignatyuk and Sokolov [28] (see Eqs. (‥42) and (‥43) below).

The corresponding cross-section formula that accounts for both the compound and the precompound effects is a straightforward extension of Eq. $(\underline{3.14})$

$$
\sigma_{\nu}\left(E, \epsilon_{\nu}\right) d \epsilon_{\nu}=\left(\frac{\lambda_{\nu}^{2}}{4 \pi}\right) \sum_{J \pi s l}\left(\frac{g_{J} T_{\nu s^{\prime} l^{\prime}}^{J}}{D_{J \pi}}\right) \sum_{p} X_{\nu}\left(n, J, I, E, \epsilon_{\nu}\right) d \epsilon_{\nu} \int_{0}^{\infty} P_{\nu}(p, J, t) d t
$$

where $\lambda_{\nu}$ is the De Broglie wave length, given by 


$$
\lambda_{\nu}=\frac{\hbar}{\mu_{\nu} v_{r e l}} .
$$

" $v_{r e l} "$ is the velocity of the relative motion of collision partners and " $\mu_{\nu}$ " is the reduced mass of the particletarget systern. In Eq. $(\underline{3.16})$, the quantity $D_{J \pi}$ is given by,

$$
\dot{D}_{J \pi}=\sum_{\nu I \pi p} \int_{\epsilon_{\nu}} X_{\nu} d \epsilon_{\nu} \int_{0}^{\infty} P_{\nu} d t
$$

and was introduced to ensure that the quantity inside the brackets has the meaning of a branching ratio, similar to the factor $\omega_{\nu}(p-1, U) / \omega(p, E)$ in Eq. $(\underline{3.11})$.

A useful approximation to deal numerically with the above expression is

$$
\frac{P_{\nu}(n, J, t)}{\omega(n, J, E)}=\frac{P_{\nu}(n, t)}{\omega(n, E)}
$$

This approximation enables the direct use of Eq.(3.14) and describes the assumption that all spin states in the composite system are equally populated during the equilibration process.

Noting that the transmission coefficients are independent of the particle-hole indices, gives

$$
\sigma_{\nu}\left(E, \epsilon_{\nu}\right) d \epsilon_{\nu}=\left(\frac{\lambda_{\nu}^{2}}{4 \pi}\right) \sum_{J \pi s l} g_{J} T_{\nu s^{\prime} l^{\prime}}^{J} \frac{d \epsilon_{\nu}}{D_{J \pi}} \sum_{s^{\prime} l^{\prime}} T_{\nu s^{\prime} l^{\prime}}^{J} \Omega_{\nu}(I, E, U)
$$

where

$$
\Omega_{\nu}(I, E, U)=\sum_{p} C_{\nu}(p, E) \rho_{\nu}(p-1, h, I, U)
$$

with

$$
C_{\nu}(p, E)=\int_{0}^{\infty} \frac{P_{\nu}(p, h, t)}{\omega(p, h, E)} d t
$$

Eq.(3.18) is the basis for cross-section evaluation in the TNG model.

If instantaneous equilibration is assumed, the above equation reduces to the usual Hauser-Feshbach formula. This arnounts to replacing the integral " $\int_{0}^{\infty} P_{\nu}(p, h, t) d t$ " by " $\omega(p, h, E)$ " and the sum " $\Sigma_{p} \rho_{\nu}(p-$ $1, h, I, U)$ " by the corventional level density, " $\rho_{\nu}(I, U)$ ".

For finite equilibration time, Eq. $(\underline{3.18})$ explicitly accounts for the spin distribution in the preequilibrium stage.

Other features of the reaction processes which are accounted for in the consistent pre-equilibrium plus Hauser-Feshbach model used in TNG are the so-called "precompound effect", which we describe in the next section, and the consistent definition of pairing correlations corrections to the level density functions, which are defined in Sec.III.4. 


\section{III.3 THE PRECOMPOUND EFFECT AND THE LEVEL DENSITIES}

Following the emission of the first particles in the direct processes, the residual levels are populated with certain spin distributions. These spin populations are different for the compound and the precompound components; the precompound processes tend to shift spin populations toward lower spins, as one can see in Fig.1 [29].

This "precompound effect" is not large at low outgoing particle energies, but for increasing incident energies the chance of higher outgoing particle energies becomes bigger and the spin-shift effect is expected to increase too. In addition, calculations for tertiary reactions such as $(n, n \alpha)$ are quite sensitive to changes in spin populations $[29,30,31]$.

Therefore, to complete the model description one must correct the level density definition to account for the precompound effect in all energy regions in a consistent manner with the compound stage description.

In the following sections we show how this can be accomplished by using an adequate formulation for the effect of pairing correlations in the pre-equilibrium stage

\section{III.3.1 Spin Distribution and Level Density in the Pre-Equilibrium Stage}

In pre-equilibrium calculations one usually makes use of the so called particle-hole (ph) state density functions, which are the functions that describe the density of sp-states for a given exciton class of $C N$-states, with explicit dependence on "p" and " $h$ ". In addition, some calculations also require the corresponding spin distributions.

In the P-E stage, the ph state-density function for the one-fermion system, $\omega_{1}(p, h, E)$, is given by the approximate formula of Williams [풀,

$$
\omega_{1}(p, h, E)=\frac{g^{(p+h-1)}\left(E-A_{p, h}\right)^{(p+h-1)}}{p ! h !(p+h-1) !}
$$

where $A_{p, h}$ is a factor to account for the "blocking" of fermion states with occupation numbers bigger than one, according to Pauli's exclusion principle,

$$
A_{p, h}=\frac{1}{4 g}\left(p^{2}+h^{2}+p-3 h\right) .
$$

The corresponding level-density function is, therefore,

$$
\rho_{1}(p, h, E, J)=\omega_{1}(p, h, E) R(n, J)
$$


where the spin distribution function for ph state-densities, in the precompound stage, is given by the expression of Feshbach et.al. [1]

$$
R(n, J)=\frac{(2 J+1)}{2(2 \pi)^{1 / 2} \sigma_{F}^{3}(n)} \exp \left[-\left(J+\frac{1}{2}\right)^{2} / 2 \sigma_{F}^{2}(n)\right]
$$

where $J$ is the spin of the nucleus and $\sigma_{F}^{2}(n)$ is the spin-cutoff factor for $C N$-states belonging to a given exciton class. The function $R(n, J)$ describes the nuclear system as a fully degenerate Fermi gas with only one type of fermions (one-fermion model), as originally proposed by Bethe [32], and satisfies the normalization condition,

$$
\sum_{J}(2 J+1) R(n, J) \approx 1
$$

The sum of the one-fermion ph-state-density function over all spins and exciton numbers,

$$
\rho_{1}(E)=\sum_{p, J}(2 J+1) \omega_{1}(p, h, E) R(n, J)
$$

can be compared with the total level-density function of the one-fermion system, given by, [25,32]

$$
\rho_{1}(E)=\frac{\exp \left[2(a E)^{1 / 2}\right]}{(48)^{1 / 2} E}
$$

in the asymptotic limit where the sum over the number of excited particles can be replaced by an integral over $k$, with $k=p=h$ (high excitations energies). In Ref. [33] it is shown that the agreement between these two functions is excellent.

To obtain a consistent description of the spin populations along the whole equilibration process these "one-fermion" results rnust also be consistent with the usual "two-fermion" level density functions used in the Hauser-Feshbach calculations. As we will see in the next section, the two-fermion level densities are formulated in a manner which takes into account the influence of pairing correlations on the single particle energy spectrum.

Therefore, an important aspect to be considered is the effect of the pairing correlations on the "onefermion" level density function. To address this point, one notices that in the original proposal of Feshbach et.al. [1] $\sigma_{F}^{2}(n)$, in Eq. ( $\left.\underline{3.22}\right)$, is defined in the usual manner [1,20]

$$
\sigma_{F}^{2}(n)=n \sigma_{1}^{2}=n c T / \tilde{n}
$$

where $\tilde{n}$ is the most probable exciton number as a function of the excitation energy, given by 


$$
\tilde{n}=\left(\frac{g E}{2}\right)^{1 / 2}
$$

$c$ is a parameter related to the nuclear moment of inertia and $\tau$ is the nuclear absolute temperature.

For high excitation energies,

$$
(g E)^{1 / 2} \gg 1
$$

the nuclear temperature is given by,

$$
\tau \approx(E / a)^{1 / 2}
$$

where, the Fermi gas parameter, " $a$ ", and the total single-particle level spacing, " $g$ ", are related by,

$$
a=\frac{1}{6} \pi^{2} g
$$

Therefore, using the approximations [ $[\underline{1}]$,

$$
c \approx \frac{A^{5 / 3}}{90} \mathrm{MeV}^{-1} \quad a \approx \frac{A}{8} \mathrm{MeV}^{-1} \quad g \approx \frac{3 A}{4 \pi^{2}} \mathrm{MeV}^{-1}
$$

one obtains,

$$
\sigma_{F}^{2}(n) \approx\left(\frac{8 \pi}{90 \sqrt{3}}\right) n A^{2 / 3}=0.161 n A^{2 / 3}
$$

Therefore, " $\sigma_{F}^{2}(n) "$ depends almost linearly on $n$ and is not dependent of $E$. Consequently, this definition cannot account for pairing correlations $[\underline{20}]$.

To circumvent this problem, TNG uses a more suitable definition for the spin-cutoff parameter in Eq. (3.22), to describe the P-E stage, which is explicitly dependent on $E$ and is consistent with the description of the pairing correlations in the $C N$-stage. This definition will be presented below in Sec.III.4.

\section{III.3.2 Level Density for the Compound Nucleus}

For calculations in the compound stage TNG makes use of the H-F formalism with level densities given by the Gilbert-Carneron (GC) formula. [27]

In the GC-model the high energy level-density function is that of the fully degenerate fermion gas with two types of fermions ("particles" and "holes") [25,27]. 


$$
\rho_{2}(E)=\frac{\exp \left[2\left(a E^{\prime}\right)^{1 / 2}\right]}{3(32)^{1 / 2} \sigma a^{1 / 4} E^{15 / 4}},
$$

where the index " 2 " refers to the number of fermion types in the model. Here, " $\sigma$ " is the spin cut-off parameter as defined in Sec.III.4.2, and $E^{\prime}$ is the effective excitation energy given by

$$
E^{\prime}=E-E_{0}-E_{P}-E_{s} .
$$

In Eq.(‥28) $E_{0}$ accounts for the odd-even effect and is often referred to as the pairing correction parameter, $E_{P}$ accounts for the Pauli-blocking feature of the many-fermion systems, and $E_{s}$ is an additional shift parameter, as explained in Sec.III.4.1 below.

The low energy portion of the composite formula corresponds to the constant temperature model

$$
\rho_{\nu}(E)=\frac{e^{\left(E-E_{0}\right) / T}}{T}
$$

and is normalized to the equation ( $\underline{3.27})$, so that both fit continuously with continuous derivatives.

Apart from the incident energy $E$, only the Fermi gas parameter " $a$ " and the pairing correction parameters are necessary as numerical input for the level density function. The parameters of the constant temperature part are directly determined by the TNG code, using a smooth connection between the discrete levels and the Fermi gas part of the level spectrum. The discrete levels and the continuum are constrained to overlap at the energy " $E_{x}$ ", which is determined empirically.

To establish the consistency between equations ( $\underline{3.24})$ for the pre-equilibrium stage and (3.27) for the compound stage, TNG uses a pairing correction term for Eq.(3.19), which we present in the following section.

\section{III.4 THE PAIRING CORRECTIONS}

In the mean-field theory [34], the nuclear system is described as a set of nucleons "dressed" by their average interactions with the other nucleons. The resulting "effective" particles are called "quasi-nucleons" and their dynamical properties are determined self-consistently. 
Under the mean-field description, the pairing correlations (among quasi-particles) result from residual interactions similar to those which produce the decay of the intermediate nuclear system in nuclear reactions. These correlations lower the sp-levels and produce an energy gap in the sp-spectrum in comparison with the non-paired states. This gap is the origin of a pairing correction term which is a displacement of the total excitation energy, corresponding to the sum of sp-displacements for all sp-excitations close to the Fermi surface.

To fully describe the pairing correlations one must work with the self-consistent formalisms of BCS $[35,36]$ or Gorkov-Bogoliubov [37]. These theories give a detailed account of the single-particle level structure of the superfluid/superconducting many-fermion systems and are the basic tools for their description.

The exciton model is compatible with the mean-field description inasmuch as the "particle" and the "hole" states which define each exciton can also be considered as quasi-particles, at least approximately. For instance, with regard to Coulomb interactions among protons, each "particle"-state can be viewed as a positive charged "island" with dynamical properties locally modified by its interactions with the surrounding negative charge, resulting from the "holes" of other excitons. And vice-versa, the "holes" can be seen as a negative charge "island" modified by its interaction with the surrounding positive particles.

In this way, in the case of protons, "particles" and "holes" are also dressed by their interactions with the environment and their phenomenological description includes this effect. Similarly, the phenomenological description of neutrons and other nuclear particles is assumed to describe the dynarnics of the corresponding dressed particles.

On the other hand, to describe the pairing correlations in a simplified way, using the exciton model, a "uniform pairing" model (UPM) has been devised, in which the single-particle states are double degenerate and the energy levels are equidistant from one another with spacing " $2 / g$ ". Therefore, the state density is twice the corresponding value in the equidistant spacing model and the Fermi level is not altered [25,32,38].

In the UPM the most important dynamical consequences of the self-consistent pairing models, such as the single-particle pairing "gap" and the determination of the Fermi energy, are treated phenomenologically. All the other features of the exciton roodel, including the residual average interactions anong nucleons (Optical Potential), are kept unaltered. 


\section{III.4.1 The Particle-Hole State Density in One-Fermion Model}

Using the UPM approximation and the BCS approach to pairing in many-fermion systems, Ignatyuk and Sokolov [28] deduced an expression for the pairing correction and Moretto [38] an expression for the minimum energy (threshold), $U_{t h}$, to excite particles close to the Fermi surface. TNG uses these results and a modified Pauli blocking term, $B,[\underline{19,20}]$ which is consistent with the expression for the density of paired states close to the Fermi surface.

The resulting effective state density is

$$
\frac{1}{g_{e f f}}=\frac{1}{g} \sqrt{1+\left(\frac{2 g \Delta}{n}\right)^{2}}
$$

and the corrected Pauli Blocking term is a natural extension of Eq.(3.20),

$$
B=\frac{1}{4 g_{e f f}}\left(p^{2}+h^{2}+p-3 h\right)=A_{p, h} \sqrt{1+\left(\frac{2 g \Delta}{n}\right)^{2}} .
$$

The threshold energy to set up an excited system with $n$ excitons, which includes pairing correlations among quasi-particles near the Fermi surface, is given by

$$
U_{t h}=\frac{g}{4}\left(\Delta_{0}^{2}-\Delta^{2}\right)+\left(\frac{n^{2}}{4 g}\right) \sqrt{1+\left(\frac{2 g \Delta}{n}\right)},
$$

where $\Delta_{0}$ is the pairing-gap in the ground-state and $\Delta$ is the pairing-gap in the excited state, for the intermediate nuclear system.

In the uniform pairing model (UPM) the threshold energy $U_{t h}$ can be approximated by the following simple numerical fit $[\underline{19}]$

$$
\frac{U_{t h}}{C}= \begin{cases}3.23\left(n / n_{c}\right)-1.57\left(n / n_{c}\right)^{2} & \text { if } \quad\left(n / n_{c}\right) \leq 0.446 \\ 1+0.627\left(n / n_{c}\right)^{2} & \text { if } \quad\left(n / n_{c}\right)>0.446\end{cases}
$$

where $n_{c}$ is the most probable quasi-particle number at the critical temperature given by

$$
n_{c}=2 g T_{c} \ln 2=\left(\frac{\ln 16}{3.5}\right) g \Delta_{0}
$$

and $C$ is the so called "condensation energy", which measures the lowering of the ground-state energy of a many-body system, due to the formation of a "boson condensate" of Cooper pairs [ $\underline{35}]$. In the uniform pairing model the condensation energy is given by

$$
C=\frac{g \Delta_{0}^{2}}{4}
$$


For excitation energies below $U_{t h}$ the spin cut-off pararneter vanishes and, consequently, the leveldensity function vanishes too. Therefore, no composite nucleus with excitation energy less than $U_{\text {th }}$ can exist. This constraint is applied in subroutine "pairl", in the source file "tngl.f", after the definition of $U_{t h}$ using Eq. (3.33)

The pairing gap for the excited system, $\Delta(U, n)$, can also be approximated by the following simple fitted function

$$
\frac{\Delta}{\Delta_{0}}= \begin{cases}0.996-1.76\left(n / n_{c}\right)^{1.60}(U / C)^{-0.68} & \text { if } U \geq E_{p h} \\ 0 & \text { if } U<E_{p h}\end{cases}
$$

where $U$ is the excitation energy and $E_{p h}$ is the energy of the pairing phase transition [39], that is, the minimum energy necessary to set up a system of " $p$ " particles and " $h$ " holes, taking into account the pairing-energy gap in the sp-levels close to the Fermi surface,

$$
E_{p h}=C\left[0.716+2.44\left(n / n_{c}\right)^{2.17}\right] \quad \text { for } n / n_{c}>0.446
$$

TNG also uses a "back-shift" parameter, $S$, similar to the one introduced by Dilg et.al. [40], which accounts for the experimentally observed behavior of the low-lying discrete levels (non-uniformity near the Fermi surface) and partially compensates for all possible deficiencies of the simplified pairing model.

The final corrected expression used in TNG for the density of states in the pre-equilibrium stage, including Pauli blocking and pairing correlations, is

$$
\omega_{1}(p, h, U, P+B+S)=\frac{g^{n}(U-P-B-S)^{n-1}}{p ! h !(n-1) !},
$$

where $P$ is the pairing correction calculated in accordance with the BCS model.

$$
P(U, n)=\frac{g}{4}\left(\Delta_{0}^{2}-\Delta^{2}\right)
$$

The parameter $\Delta_{0}$ is determined, in the "generalized superfluid model" (GSM) of Ignatyuk et.al. [녈], by the following dependence with the target mass, [1ㅣ]

$$
\Delta_{0}=\frac{12}{\sqrt{A}}
$$

in subroutine "para", in the file "dntrnpr.f".

Another definition is used in subroutine "pair1", in which the relation between $\Delta_{0}$ and the condensation energy, "C" defined in Eq. $(\underline{3.35})$, is directly used. In this case, "C" is entered as the input parameter "QC" (the 2nd. entry of Card 14 of unit-60), which can be negative if the back-shifted fermi gas model is used. If "QC" is negative, the spin cut-off function given by the expression of Reffo and Herman, Ref. [ㅍ], 


$$
\sigma^{2}(U, n)=0.28 \tilde{n} A^{2 / 3}
$$

where $\tilde{n}$ is the most probable exciton number, which is a function of " $U$ ".

\section{III.4.2 The Spin-Cutoff Parameter}

As we have seen in Sec.III.3.1, the precompound reaction calculations use the spin distribution function of the particle-hole state density given by the formula of Feshbach et.al. [1].

TNG uses a slightly modified version of Eq.(3.22) in which the spin-cutoff factor, $\sigma^{2}(U, n)$, is now explicitly written in terms of given exciton number $n$ and excitation energy $U$ of the excited nuclear states, $\underline{[1,20]}$

$$
R(n, J)=\frac{(2 J+1)}{2(2 \pi)^{1 / 2} \sigma^{3}(U, n)} \exp \left[-\left(J+\frac{1}{2}\right)^{2} / 2 \sigma^{2}(U, n)\right]
$$

The spin-cutoff factor used in TNG is the general expression of Ignatyuk and Sokolov [28], which takes into account the pairing correlations in the UPM approximation,

$$
\sigma^{2}(U, n)=2 g<m^{2}>\int_{0}^{\infty} f(\epsilon)[1-f(\epsilon)] d \epsilon
$$

This expression includes the dependence on the excitation energy of the $C N$-system, as required for the compound cross sections calculations, and a consistent dependence on the exciton number in the preequilibrium stage. $[\underline{1,4,20}]$

In Eq. (3.43) $g$ is the single-particle state density, $\left\langle m^{2}\right\rangle$ is the mean square of the projections of the spins of particles and holes on the z-axis of the nucleus and $f(\epsilon)$ is the Fermi probability distribution function given by

$$
f(\epsilon)=\frac{1}{1+\exp [(E-\xi) / T]}
$$

which is the probability of occupancy for an sp-level with energy $\epsilon$ by a fermion, that is, the probability that this state is occupied by a particle is " $f(\epsilon)$ " and the probability that it is empty is given by " $(1-f(\epsilon))$ " [녀].

The parameter $T$ is the absolute temperature of the nuclear system, $E$ is the energy of the sp-levels given by

$$
E=\left(\epsilon^{2}+\Delta^{2}\right)^{1 / 2}
$$


and the number of particles and holes obeys the UPM condition,

$$
p=h=n / 2 \text {. }
$$

The quantity $\xi$ is a Lagrange multiplier to constrain the number of quasi-particles to be equal to the total number of particles of the systern. In the BCS-model $\xi$ coincides with the thermodynamic chemicalpotential parameter and with the Fermi-energy of the system [34,38].

The values of $\Delta, \xi$ and $T$ can be obtained numerically from a set of pairing equations for each combination of $g, \Delta_{0}, U$ and $n$. Therefore, the dependence of $\sigma^{2}$ in $(U, n)$ in Eq.(3.43) is implicit in the functions $\Delta, \xi$ and $T$.

In TNG Eq.(3.43) for the spin-cutoff term is replaced by a simple fitted expression given by

$$
\sigma^{2}(U, n)=(\ln 4)\left(\frac{n}{n_{c}}\right)\left(\frac{U-U_{t h}}{U}\right)^{x}
$$

where the exponent is a function of " $\left(n / n_{c}\right)$ ", given by

$$
x=-0.413+1.08\left(\frac{n}{n_{c}}\right)^{1 / 2}-0.226\left(\frac{n}{n_{c}}\right) .
$$

Here, the minimum excitation energy, $U_{t h}$, is given by Eq. $(\underline{3.33})$.

\section{III.5 FINAL COMMENTS AND USEFUL INPUT HINTS}

The TNG code uses the model described in the previous sections, which allows a consistent description of intermediate excitations of the equilibrating nuclear system. This formalism permits the calculation of binary and tertiary reaction cross sections for processes in which the residual nucleus has discrete levels with known spins and parities.

TNG is a relatively small code. It was originally designed for small computers (PDP-10 and VAX) with limited memory and speed capabilities. Presently it is implemented as part of SAMMY, and its future implementations shall not take this kind of restrictions into account. It has been continuously improved and used at ORNL for the evaluation of neutron-induced reaction cross sections for the ENDF/B series since $1972[\underline{10}]$.

It can predict neutron, proton, and alpha-particle production cross sections, as well as, the total, elastic and nonelastic cross-sections and spectra from neutron-induced reactions. 
The Hauser-Feshbach part of the numeric procedure is multi-step, but the angular distribution can be calculated only for the binary step. The pre-equilibrium part is single-step and the angular distribution is calculated on the basis of a relaxation of the random phase approximation used in the H-F formulation.

The code also calculates the secondary-particle emission spectra, the angular distributions of the outgoing particles in the first pre-equilibrium step and the cross-sections for production of isomeric states.

Another feature of TNG is the inclusion of spherical optical model module, with 10 sets of default options for the parametrization of the potential. One possibility here corresponds to using the Optical Potential obtained with code GENOA.

Some useful hints on the input data of TNG are given in the following. The user should be careful here because the definitions of entries in the input fles, unit-60 and unit-63, are interconnected.

The internal settings for the Optical Potential correspond to negative values $(-1,-2, \ldots,-9)$ of the variable "IOP" (corresponding to the second, third and fourth entries of Card 5 in unit-60) and "NNUC", the sixth entry of Card 4 of unit-63. The potential from GENOA corresponds to the value "1" either for "IOP" or "NNUC". (see Appendix A for details).

The information on the level structure of the residual nucleus, for binary reactions, must always be input. It uses the parameter "NLVL1", in Card 10 of unit-60, and the parameters "ENU", "AIT" and "AP", in Card 11 of unit-60 (see details in Sec. A.1).

Similar information must also be input for the evaluation of the spectra of all tertiary reactions. In this case, the level information is entered in the parameters "NLEV" (the first entry in Card 4 of unit-63) and "ELEV", "SPIN" and "PAR", in Card 6 of unit-63 (see details in Sec. A.2).

The binary reactions include the following three types,

$$
\left(n, n^{\prime}\right),(n, p), \text { and }(n, a),
$$

and its number is determined by the parameter "NBS" (first entry in Card 4 of unit-60). Here " $a$ " stands for an alpha particle in the final channel.

The input for tertiary reactions, fission and radiative absorption are entered in unit-63 and include the following processes,

$$
(n, f),(n, g)
$$

and

$$
(n, 2 n), \quad(n, \pi p), \quad(n, n a),(n, n f), \ldots,(n, 4 n), \text { etc. }
$$


where $g$ stands for gamma-emission and $f$ for the fission process.

The Gamma-ray emission spectrurn for each reaction can also be calculated by setting the parameter "RAD" equal " 1 " (second entry in Card 8 of unit-60) and "NTPCRD" bigger than "0" (second entry in Card 4 of unit-63).

The cross-sections for the fission channel can be calculated by setting the parameter "FISS" bigger than "0" (third entry in Card 8 of unit-60). Only in this case the Cards 12 to 16 of unit-63 should be present, to describe the features of the fission barrier as, e.g., the number of "hurnps" and the level structure of each hump. The double-humped model for the fission barrier is the default and the single-humped model is used if the second barrier has height zero (see Sec. A.2).

The sequence of calculations is determined by the parameter "IMORE" (the second entry in the last Card, no. 17, of unit-63); which specifies if the code should return to binary step or continue in the tertiary step to evaluate other reactions.

A detailed description of the two input fles for TNG, unit-60 and unit-63, can be found in Appendix A. 


\section{IV - THE GENOA CODE}

GENOA [ $[$ ] is based on the Optical Model for nuclear reactions and was developed by Francis $\mathrm{G}$. Perey, at the Oak Ridge National Laboratory in the late 60's. It performs the numerical "search" for one average set of theoretical parameters to define a global Optical Potential, which is able to describe simultaneously several sets of experimental data. Normally this code is used along with TNG to evaluate nuclear data in the unresolved-resonances energy region.

The Optical Potential model [ $\underline{3}$ ] deals with elastic and non-elastic processes in which the interaction between incident and target nuclear systems can be described, approximately, by an average central-potential with a simple parametrization.

As a simple rule one may say that for nucleon (neutron, proton) induced reactions, with incident energies in the unresolved-resonances region and for medium and heavy target nuclei, the Optical Model applies. [6] In this case, the equilibration process probably produces a final $C N$ state with many excited single-particle states, because the energy spread of the incident beam is probably much greater than the mean level spacing of the target nucleus. Therefore, the experimentally observed reaction parameters will not depend critically on the detailed structure of the target, but only on its general average properties.

\section{IV.1 DESCRIPTION OF THE OPTICAL POTENTIAL IN THE GENOA CODE}

The Optical Model for nuclear reactions follows the basic definitions of the Shell Model for nuclear structure [6]. That is, it assumes, that the exact many-body interaction between the incident and target nuclear systems can be replaced by an average phenomenological "Optical Potential", $V(\mathbf{r})$, where $\mathbf{r}$ is the relative distance between the centers-of-mass of the two systems.

Although the Optical Potential can be derived from "first principles", using adequate definitions of the direct and compound processes [1,7], in practice it is obtained by numerically fitting a given class of reaction cross-sections, with a given range of incident energies and target masses.

The potential " $V(\mathbf{r})$ " is generally considered complex to account for the possible channels in which the intermediary $C N$ is formed, called "cornpound channels". 
In the Optical Model the compound channels include all reactions in which the final channel is different from the entrance channel plus the "compound elastic channel", in which the $C N$ decays back into the entrance channel itself. On the other hand, a purely real Optical Potential could only account for the so called "shape elastic" processes, i.e., the elastic reactions in direct processes, without nucleon transfer reactions.

More specifically, the potential " $V(\mathbf{r})$ " can account for both compound and direct processes and in both cases the description is approximate. In the compound-reaction case the formation of the $C N$ is described as an absorption, using the imaginary part of the potential. In the direct-reaction case no inelastic processes can be described but only the shape-elastic scattering. The possible modifications of the target are not described by the model. Instead, the target is assumed to remain in its initial state, with the sole function of producing the average reaction potential. []

To consider nucleon transfer reactions, as the "pick-up reactions" and the "direct knock-out reactions", as well as the fission channel, the Model must be adapted, by using a multi-step description and the principle of detailed balance. This adaptation of the OM is implemented in TNG. In this way, using the multi-step formalism, the possible modifications of the target are inserted by hand, at each step.

\section{IV.1.1 Formal Definitions in the Optical Model}

Initially, we present here some general concepts on the nucleon-nucleon interactions to motivate the formal definitions of the interacting potential, which are given in the following.

Generally speaking, it is experimentally observed that the nucleon-nucleon forces are short-ranged and have a saturation property. They are large and repulsive when nucleons are close together, about 0.7 fm of relative distance, and become attractive at larger distances. They are very strong, when compared to the Coulomb interaction, but can only be felt if the nucleons are not far apart.

Above a few Fermis of distance the nuclear forces become negligibly small and, in the opposite direction, they become almost infinitely repulsive at about $0.4 \mathrm{fm}$. Furthermore, due to the quantum principles of Pauli's Exclusion and Incertitude, the nucleons stay rather far apart within the nucleus, about $2.4 \mathrm{fm}$ on the average, and do not interact directly with each other, but rather with an average field produced in a self-consistent manner by its simultaneous interaction with all other nucleons in the system. 
Therefore, the bounded nucleons within the nuclear system move almost freely but constrained to a rather small space region. This gives rise to a first approximation description of the nuclear system as a Ferni gas, on the microscopic point of view, and also as a liquid drop for the description of the bulk properties of all nucleons as a whole. Another consequence of these features is that the distribution of nucleons in the nuclear volume, the "nucleon density" or "baryon density", follows closely the shape of the nuclear potential itself.

This general dynamical state defines an average nuclear force which is large within the nucleus and decreases rapdly toward zero in the nuclear boundary region. This region is defined as the "surface" of the nucleus and is better described as diffuse instead of sharp [3,34,44] . The formal definitions of the nuclear potential form-factors try to take these features into account.

In GENOA the Optical Potential is defined, as usual, by four parts named real, imaginary, spin-orbit and Coulomb. The real and imaginary parts of the potential account for the interaction with the strong, short-range nuclear forces. These "nuclear" terms are divided into "volume" and "surface" parts to account for the average features of the dynamics of the nucleons in the nuclear system, as described above.

The Optical Model calculations deal with the physical definitions and constraints of a scattering process, which is described by the solution of the a time-independent Schroedinger equation,

$$
\frac{-\hbar^{2}}{2 m} \nabla^{2} \psi+V(\mathbf{r}) \psi=E \psi
$$

with $V(\mathbf{r})$ given strictly by the cornplex Optical Potential defined below. No other angular-momentum dependent interaction is assumed beyond the centrifugal potential and the spin-orbit coupling.

The potential $V(\mathbf{r})$ is defined by a sum of terms;

$$
V(\mathbf{r})=V_{R}(r)+i W(r)+V_{\mathrm{so}}(r)+V_{c}(r)
$$

where the algebraic expressions for the various parts, as defined in GENOA, are the following:

The Real Part consists of the volume and surface components of a nuclear potential associated with elastic scattering, the so called "shape-elastic" process, in the interaction of the incident particle with the nuclear forces,

$$
V_{R}(r)=U f\left(r, R_{R}, a_{E}, a_{R}\right)+U B g\left(r, a_{B}\right)
$$

The Imaginary Part consists of the volume and surface components of an non-elastic, "absorption" or "compound", process in the interaction of the incident particle with the nuclear potential, 


$$
W(r)=W_{S} h\left(r, R_{I}, a_{I}\right)+4 a_{I} W_{D} h\left(r, R_{I}, a_{I}\right)
$$

The Spin-Orbit Part accounts for the coupling between the Spin, "s", and the Orbital angular momentum, "l", of the scattered particle, in its interaction with the nuclear potential,

$$
V_{\mathrm{So}}(r)=\left(V_{\mathrm{SO}}+i W_{\mathrm{So}}\right) h\left(r, R_{\mathrm{so}}, a_{\mathrm{SO}}\right) \mathbf{l} \cdot \mathrm{s}
$$

and the Coulomb part accounts for the Coulomb interaction of the scattered particle with the rest of the nuclear systern. This part is given by the potential of a uniform distribution of radius $R_{c}$,

$$
V_{c}(r)=\left\{\begin{array}{lll}
\left(\frac{3}{2}-\frac{r^{2}}{2 R_{c}^{2}}\right) Z_{p} Z_{t} \mathrm{e}^{2} / R_{c} & \text { for } & r \leq R_{c} \\
Z_{p} Z_{t} \mathrm{e}^{2} / r & \text { for } & r \geq R_{c}
\end{array}\right.
$$

where $Z_{p}$ and $Z_{t}$ are the projectile and target charges, respectively, and "e" is the electric charge of the proton.

Usually $V_{c}$ is rewritten as,

$$
V_{c}(r)=\left\{\begin{array}{lll}
\left(\frac{3}{2}-\frac{r^{2}}{2 R_{c}^{2}}\right)\left(\frac{\eta}{k R_{c}}\right) & \text { for } & r \leq R_{c} \\
\left(\frac{2 R_{c}}{r}\right)\left(\frac{\eta}{k R_{c}}\right) & \text { for } & r \geq R_{c}
\end{array}\right.
$$

where the Coulomb parameter, $\eta$, is given by,

$$
\eta=\frac{Z_{1} Z_{2} e^{2}}{(\hbar c)(v / c)}
$$

and " $k$ " is the incident particle wave-number in the CM reference frame.

The other features are the various nuclear-force ranges,

$$
R_{i}=r_{0 i} A^{1 / 3}
$$

where $i=" R^{\prime \prime}$ (real)," $I^{\prime \prime}$ (imaginary) or "SO" (spin-orbit), and the form-factors for the corresponding nuclear wells.

The real-surface well is considered to have a Gaussian form-factor,

$$
g(r, a)=\exp \left(-r^{2} / a^{2}\right)
$$

and the real-volume well is taken with a Woods-Saxon form [44], 


$$
f\left(r, R, a_{1}, a_{2}\right)= \begin{cases}1 /\left[1+\exp \left((R-r) /\left(a_{1}\right)\right)^{2}\right] & \text { for } r \geq R_{c} \\ 1 /\left[1+\exp \left((R-r) /\left(a_{2}\right)\right)^{2}\right] & \text { for } r<R_{c}\end{cases}
$$

The imaginary-surface and the Spin-Orbit terms are taken as the derivatives of a Woods-Saxon form. Therefore, the function $h$ in Eq.(4.4) has the following general definition,

$$
h(r, R, a)=1 /\left[1+\exp \left(\frac{R-r}{a}\right)^{2}\right] .
$$

Usually GENOA has a set of nearly 10 searching-parameters to define the average global potential. These parameters are defined in a way that permits greater flexibility, when fitting several data sets, than the direct fitting of the various physical parameters.

In this way, this structure enables the introduction of specific functional dependencies of the potentialparameters with more than one search-parameter. For example, for a given potential-parameter a second search-parameter can be defined to specify other functional dependencies, on the energy of the incident particle, on the mass number of the target, etc.

These two numeric procedures are matched in a subroutine called "BUFFEG", where the definitions of the functional relationship among the diverse parameters is done. In the next section we give some formal details of the numerical procedure adopted in GENOA, followed by a description of the subroutine "BUFFEG".

\section{IV.2 NUMERICAL PROCEDURE}

GENOA uses two independent flows of numeric calculation []]. In one flow the code performs numerical fitting procedures to determine the searched parameters, the so called "central" parameters. In the other numeric flow, the code performs Optical Model calculations in which the physical definitions and constraints of the problem are defined.

In terms of the segments of SAMMY, the OM calculations are performed by "samgit", while the numerical fitting is performed by "sammpw", which is also called by other parts of the code SAMMY, as we have seen in Sec.II.1. . 


\section{IV.2.1 Numerical Integration of the Schroedinger Equation}

Either in GENOA or TNG, the solution of Eq.(4.1) is performed with the help of an iterative procedure in which the eigenfunction is determined by numerical integration. In this procedure the expression to be integrated is obtained with the method of finite differences applied directly to the Schroedinger equation. This procedure is implemented in the subroutines "ITEGL" and "SSCMAT" in GENOA and subroutine "CHAPSO" in TNG, and the final results, in both cases, are the Optical Model cross-sections for the "shape-elastic", "compound" and "total" processes.

The procedure is based on the following expression for the definition of the cross-section, $\sigma_{c}$, to form a compound nucleus with excitation energy " $E$ " by an incident particle with orbital angular momentum "l",

$$
\sigma_{c}(E, l)=\frac{\pi}{k^{2}}(2 l+1)\left(1-\left|\eta_{l}\right|^{2}\right)
$$

where " $k$ " is the wave-number of the incident particle and $\eta_{l}$ is the scattering matrix element for the reaction process between the given initial and final states of the incident particle, " $|i\rangle$ " and " $|f\rangle$ ", in which the orbital angular momentum " $l$ " is conserved. The term,

$$
T_{l}(E)=\left(1-\left|\eta_{l}\right|^{2}\right)
$$

is the transmission coefficient for the dynamical process of formation of the cornpound system with the given $(E, l)$-initial channel.

In this case, $\eta_{l}$ is given by $[\underline{3,50}]$

$$
\eta_{l}=\frac{f_{l}-\Delta_{l}+i s_{l}}{f_{l}-\Delta_{l}-i s_{l}} \exp \left[2 i \xi_{l}\right]
$$

$f_{l}$ is given by the logarithmic derivative,

$$
f_{l}=R\left[\frac{1}{u_{l}}\left(\frac{\partial u_{l}}{\partial r}\right)\right]_{r=R}
$$

and the functions $\Delta_{l}$ and $s_{l}$ are the real and imaginary parts in the following expression,

$$
\Delta_{l}+i s_{l}=R\left[\frac{1}{u_{l}^{(+)}}\left(\frac{\partial u_{l}^{(+)}}{\partial r}\right)\right]_{r=R} .
$$

In Eqs.(4.15) and (4.16) the logarithmic derivatives are calculated at the nuclear boundary radius, $r=R$.

In Eq.(4.15) $u_{l}$ is the eigen-solution of the Schroedinger equation given by,

$$
u_{l}(r)=i^{l+1} \frac{[(2 l+1) \pi]^{1 / 2}}{k}\left(u_{l}^{(-)}(r)-\eta_{l} u_{l}^{(+)}(r)\right)
$$


where " $u_{l}^{(-)}(r)$ " and " $u_{l}^{(+)}(r)$ " are the "in going" and "outgoing" spherical waves, respectively, which define a complete set of independent solutions of the radial Schroedinger equation. The paranneter " $\xi_{l}$ " in Eq. (4.14) is the phase shift between the in going and the outgoing wave-functions at the nuclear boundary, defined by the expression,

$$
\exp \left[2 i \xi_{l}\right]=\frac{u_{l}^{(-)}(R)}{u_{l}^{(+)}(R)}
$$

To obtain the expression used in the iterative numerical integration we rewrite Eq.(4.1) as

$$
\begin{aligned}
-\frac{\partial^{2} u_{l}(r)}{\partial r^{2}}= & \frac{2 m}{\hbar^{2}}[E-V(\mathbf{r})] u_{l}(r) \\
& =\frac{k^{2}}{E}[E-V(\mathbf{r})] u_{l}(r)
\end{aligned}
$$

and use the method of finite differences to approximate the left hand side, as follows

$$
\frac{\partial^{2} u_{l}(r)}{\partial r^{2}} \approx \frac{1}{\Delta^{2} r}\left(u_{l}(r(i+1))+u_{l}(r(i-1))-2 u_{l}(r(i))\right)
$$

where $r(i)$ is now a discrete radial variable defined by

$$
r(i)=i \Delta r, \quad i=1,2, \ldots, N
$$

$N$ is the total number of steps in the numerical radial integration and $\Delta r$ is a small radial step given by

$$
\Delta r=\frac{R}{N}
$$

Then, we can rewrite Eq.(‥18) as

$$
-\left(u_{l}(r(i+1))+u_{l}(r(i-1))-2 u_{l}(r(i))\right)=P(\mathbf{r}) u_{l}(r)
$$

where $P(\mathbf{r})$ is a function of the OM potential given by,

$$
P(\mathbf{r})=\frac{k^{2} \Delta^{2} r}{E}[E-V(\mathbf{r})]
$$

To obtain the corresponding approximate expression for the right hand side of Eq.(4.18), we integrate both sides mumerically in the interval $[r(i-1), r(i+1)]$, using Simpson's extended method with 5 points [51]. The left hand side is constant in this interval, because it is already approximate, and gives

$$
\begin{aligned}
\int_{r(i-1)}^{\tau(i+1)}\left(u_{l}(r(i+1))+u_{l}(r(i-1))-2 u_{l}(r(i))\right) \\
=2 \Delta r\left(u_{l}(r(i+1))+u_{l}(r(i-1))-2 u_{l}(r(i))\right) .
\end{aligned}
$$


The right hand side of Eq.(‥18) becornes,

$$
\begin{aligned}
\int_{a}^{b} P(\mathbf{r}) u_{l}(r) \approx \frac{(b-a)}{12}\left[P(\mathbf{r} 1) u_{l}(r 1)+\right. & 4 P(\mathbf{r} 2) u_{l}(r 2)+2 P(\mathbf{r} 3) u_{l}(r 3) \\
& \left.+4 P(\mathbf{r} 4) u_{l}(r 4)+P(\mathbf{r} 5) u_{l}(r 5)\right]
\end{aligned}
$$

where the various points are, $a=r(i-1)=r 1, r 2=(b+3 a) / 4, r 3=(b+a) / 2=r(i), r 4=(3 b+a) / 4$ and $b=r(i+1)=r 5$. Then, assuming that the function " $P(\mathbf{r}) u_{l}(r)$ " varies slowly in the interval $[r(i-1), r(i+1)]$, one can approximate the intermediate values as,

$$
P(\mathbf{r 2}) u_{l}(r 2) \approx P(\mathbf{r} 4) u_{l}(r 4) \approx P(\mathbf{r 3}) u_{l}(r(i))
$$

Hence, Eq.(4.24) becomes

$$
\begin{gathered}
\int_{a}^{b} P(\mathbf{r}) u_{l}(r) \approx \frac{(b-a)}{12}\left[P(\mathbf{r} 1) u_{l}(r 1)+10 P(\mathbf{r} 3) u_{l}(r 3)+P(\mathbf{r} 5) u_{l}(r 5)\right] \\
=\frac{(2 \Delta r)}{12}\left[P(r(i-1)) u_{l}(r(i-1))+10 P(r(i)) u_{l}(r(i))+P(r(i+1)) u_{l}(r(i+1))\right]
\end{gathered}
$$

Then, joining the results of Eqs.(4.23) and (․․26) into the Schroedinger equation, (4.22), results,

$$
\begin{gathered}
2 \Delta r\left(-u_{l}(r(i+1))-u_{l}(r(i-1))+2 u_{l}(r(i))\right) \\
\approx \frac{(2 \Delta r)}{12}\left[P(r(i-1)) u_{l}(r(i-1))+10 P(r(i)) u_{l}(r(i))+P(r(i+1)) u_{l}(r(i+1))\right]
\end{gathered}
$$

which can be rewritten as,

$$
\begin{gathered}
\left(12 u_{l}(r(i+1))+12 u_{l}(r(i-1))-24 u_{l}(r(i))\right) \\
\approx\left[P(r(i-1)) u_{l}(r(i-1))+10 P(r(i)) u_{l}(r(i))+P(r(i+1)) u_{l}(r(i+1))\right]
\end{gathered}
$$

Equation (4.27) can then be used to find $u_{l}(r(i+1))$, given $u_{l}(r(i-1))$ and $u_{l}(r(i))$. This particular method of solving Eq.(‥18) is the Numerov's method, for linear differential equations of second order without first derivatives [드] ], and has the absolute truncation error,

$$
\frac{(\Delta r)^{6} u_{l}^{(V I)}(r)}{240}
$$

where " $u_{l}^{(V I)}(r)$ " is the fourth derivative of $u_{l}$ with respect to $r$. This finite differences equation is used in "ITEGL"(GENOA) and "CHAPSO"(TNG) to determine $u_{l}$ at the nuclear boundary region, $r=R$.

The resulting $u_{l}(R)$ is then used to determine its derivative, also using the method of finite differences, and the function $f_{l}$, in Eq.(4.15). Then, the expressions (․14) and (4.12) are used to determine the crosssections of the compound processes. 


\section{IV.2.2 Bayes' Equations}

The numerical fitting is based on the generalized least squares method, which is the same used by other segments of SAMMY [12] and is defined by a set of equations which result from Bayes' theorem on conditional probability evaluation.

This method connects the sequence of iterative parameters in data fitting by a covariance matrix of the theoretical parameters, thus assuming a "prior knowledge" of these parameters at each new iteration step. This contrasts with the simple "leasts squares". method in which no such knowledge is assumed. A detailed derivation of Bayes' equations can be found in SAMMY user's manual [14].

The basic formal result is

$$
p(P \mid D B) \propto p(P \mid B) p(D \mid P B)
$$

where $p(a \mid b)$ is the conditional probability of event " $a$ ", taking into account that event " $b$ " has occurred.

The variable " $P$ " represents the set of parameters that we want to determine, the "theoretical parameters" referred above, while " $D$ " represents the source data which we want to fit using " $P$ ".

It is not necessary that " $D$ " and " $P$ " have the same theoretical meaning, but the two sets must be functionally connected. In nuclear data analysis, " $D$ " represents the experimental data on reaction crosssections and in the GENOA code " $P$ " represents the set of central parameters which define the Optical Potential.

" $B$ " represents the other relevant information to determine " $P$ ", which characterizes the "prior knowledge" feature in the Bayes' description of the probability function.

A possible explicit expression for the Bayes' equations, as used in GENOA, is

$$
P^{\prime}=P+M^{\prime} Y
$$

where the primes indicate the updated results for a given variable in the iterative numerical procedure.

The variable $M$ is the covariance matrix for the central-parameters, $P . Y$ is the approximate first order variation of the theoretical parameters with respect to the experimental data,

$$
Y=G^{t} V^{-1}(D-T)
$$

where, $V$ is the covariance matrix for $D$, the experimental data matrix, $T$ is the matrix of theoretical ("evaluated") cross-sections and $G$ is the matrix of the partial derivatives of $T$ with respect to $P$. 
The updated values of $M$ are given in terms of theoretical cross-sections, $T$, by the equation,

$$
M^{\prime}=\left(M^{-1}+W\right)^{-1}
$$

where $W$ is the variation of the parameter covariance matrix defined as

$$
W=G^{t} V^{-1} G
$$

As we said before, the majority of these calculations are performed by the independent segment "sammpw" of SAMMY, except the non-linear corrections in $G$, for $Y$ and $W$, which are performed in the subprogram "sgenoa", which belongs to the segment "samgit". The subprogram "sgenoa" centralizes and directs all calculation flows in GENOA.

\section{IV.2.3 The Subroutine "BUFFEG"}

According to the definitions of Sec.IV.2.2 GENOA uses the set of 18 Optical Model parameters given in Tab.I.

The correspondence between the "physical" Optical Model parameters and the "numerical" centralparameters is set up in subroutine "BUFFEG", as shown in Tab.II.

\begin{tabular}{llllll}
\hline \hline \multicolumn{5}{c}{ Table - I } \\
\hline \multicolumn{5}{c}{ Optical model parameters } \\
\hline$U$ & $r_{0 R}$ & $a_{R}$ & $a_{E}$ & $B$ & $a_{B}$ \\
$W_{s}$ & $W_{D}$ & $r_{0}$ & $a_{I}$ & & \\
$V_{s o}$ & $W_{s o}$ & $r_{0 s o}$ & $a_{s o}$ & \\
$r_{0 c}$ & $N \chi^{2}$ & $\chi^{2} \sigma$ & $\chi^{2} P$ \\
\hline
\end{tabular}

The central-parameters correspond to the components of the array "xcal(i)", of dimension "ngnpar", which is defined in the subroutines "NROUTO", in the source file "/git/sgenoa.f", and "BUFFEG", in the file "/git/buffeg.f". The components of the array "xcal" are input as a guess at the beginning of the calculations and are also the parameters to be searched.

In the original definitions of GENOA the value of "ngnpar" was precisely equal to the number of central parameters to be varied. Presently, the value of "ngnpar" is bigger than this to permit the introduction of some new numeric degrees of freedom in the searching procedure. The value of "ngnpar" is directly specified by GENOA using the information in the input data file, unit-50 (file "fort.50"). The 
code counts the components of "xcal" which are going to be varied in accordance with the various options associated with the parameter "NCOMP", as explained below. The parameter "NCOMP" is defined in Card 7 of unit-50 and determines how the compound elastic cross-section magnitude for each reaction shall be included in the searching procedure.

In the original definition for the option "NCOMP" equal 1, the parameter "ADC", which is named as an abbreviation to mean "Add Compound elastic processes", was fixed and equal to 1.

To vary the magnitude of the total cornpound-elastic cross-section, simultaneously with the search for the Optical Model parameters, only the option "NCOMP" equal 2, for a given reaction, was available. In this case, the parameter "ADC" was used as a multiplicative factor to obtain the best-fit magnitude of the compound elastic cross section, $\sigma_{C E}$. Then, the energy-dependent magnitude of the compound elastic contribution was represented by the empirical relation,

$$
\sigma_{C E}(E)=A /[1+\exp [(B-E) / C]]
$$

where each parameter "A", "B" or "C", were associated with independent components of the array "xcal". This option, "NCOMP $=2$ ", has not been changed with respect to its original definition.

In the present definition of "BUFFEG", The options "NCOMP $=5,6$, or 7 " use independently variable "ADC" with values proportional to the parameters "fadc" and "fadc1", which are the two first entries of Card 1. of unit-50.

If NCOMP is equal to " 3 " or " 4 " for a given reaction, the corresponding component of "xcal" is fixed and equal to zero or "fadc", respectively.

The present possible values of "NCOMP", corresponding to the various options for the variation of "ADC", are the following,

NCOMP equal to: 1 ADC varies independently for each reaction with values close to 1 .

3 ADC has fixed value equal to zero.

4 ADC has fixed value equal "fadc" (taking "fadc" $=1$ one recovers the original "NCOMP $=1$ " case).

5 ADC varies independently with values close to "fadc".

6 ADC varies independently with values close to "fadcl".

7 ADC varies independently with values proportional to "fadc" and the proportionality factor also varies independently for each reaction. 
The definitions of "BUFFEG" are closely connected with the parameters of the Card 1 (see Appendix B ). In Card 1 the parameter "nl0" specifies the number of central parameters directly used in the definition of the O. M. Potential in subroutine "BUFFEG", while " $m 5$ " specifies the central parameters associated with the definitions of the options "NCOMP $=2$ " and "NCOMP $=7 "$ ".

Another entry of Card 1 connected with the definition of the compound elastic term is the parameter "nadc", which is defined in relation with the total number of experinental data sets in the search, parameter "kto" in Card 2 of unit-50. If "nadc" is smaller than "kto" the components of "xcal" between "n10+nadc+1" and "n10+kto" vary proportionally to each other and to the same component of "xcal", "xcal(kto+n10+4)". If "nadc" is equal to "kto", the components of "xcal" between "n10+1" and "n10+kto" may vary independently of each other, according to the rules given above.

To each central-parameter, "xcal(i)", is associated a corresponding "variation" defined by the components of the array "eps", in subroutine "NROUTO", which has dimension "ngnpar+1" and is used to define the magnitude of the central-parameters in the next iteration.

\begin{tabular}{|c|c|c|c|c|c|}
\hline \multicolumn{6}{|c|}{ Table - II } \\
\hline \multicolumn{6}{|c|}{ Optical Model Symbols $\times$ Variables Used in BUFFEG } \\
\hline \multirow[t]{7}{*}{ 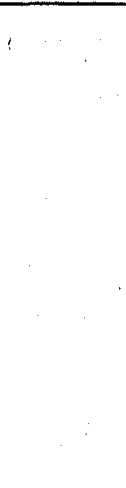 } & Potential & Imaginary & Potential & Spin & Orbit \\
\hline & $\mathrm{U}$ & $W_{S}$ & WS & $V_{\text {so }}$ & USP \\
\hline & $\mathrm{RR}$ & $W_{D}$ & WD & $W_{\text {so }}$ & WSP \\
\hline & AR & $r_{O I}$ & RI & roso & $\mathrm{RS}$ \\
\hline & ARE & $a_{I}$ & AI & $a_{\text {so }}$ & AS \\
\hline & B & & & $r_{o c}$ & $\mathrm{RC}$ \\
\hline & $\mathrm{ARB}$ & & & & \\
\hline
\end{tabular}

Other physical parameters used in the definitions of the O. M. potential in BUFFEG are,

$$
\begin{aligned}
\mathrm{E} & =\text { reaction energy in } \mathrm{MeV}(\mathrm{Lab}) \\
\mathrm{AMT} & =\text { mass of the target nucleus in AMU } \\
\mathrm{AMI} & =\text { mass of the incident particle in AMU } \\
\mathrm{ZT} & =\text { charge of the target nucleus }
\end{aligned}
$$


and

$$
\mathrm{ZI}=\text { charge of the incident particle }
$$

The default number of independent central-parameters for each optical potential parameter is given in Tab.III, where the potential parameters associated with 2 central-parameters have one of them to account for a linear dependence of the fitted optical parameter with the incident particle energy, $E$.

One example of the basic relations between central-parameters and OM-parameters in "BUFFEG" is shown in Tab.IV.

\begin{tabular}{c|l|c}
\hline & \multicolumn{1}{|c}{ TABLE - III } & \multicolumn{1}{c}{ Number of } \\
\hline Variable & \multicolumn{1}{|c}{ Description } & $\begin{array}{c}\text { Central } \\
\text { Parameters }\end{array}$ \\
\hline $\mathrm{U}$ & real volume potential magnitude & 2 \\
$R_{R}$ & nuclear radius for the real potential & 1 \\
$a_{R}$ & difusivity of the real volume potential & 1 \\
$W_{D}$ & irnaginary surface potential magnitude & 2 \\
$W_{S}$ & indaginary volurue potential Inagnitude & 2 \\
$R_{I}$ & nuclear radius for the imaginary volume and surface potential & 1 \\
$a_{I}$ & diffusivity of the imaginary volume and surface potential & 1 \\
\hline \hline
\end{tabular}

In Tab.IV, one can see that some potential parameters are defined as functions of the previous ones. For instance, $R_{\mathrm{SO}}$ and $R_{c}$ are set equal to $R_{R}$ and $V_{\mathrm{So}}$ is set equal to $U$. In addition, the parameters $W_{\mathrm{SO}}, a_{E}$ and $B$ are usually taken as equal to zero. Therefore, the magnitude of the real surface potential, $B_{S}=B U$, is usually zero too.

The user can modify the subroutine "BUFFEG" to introduce new physical variables or to define specific dependencies of the existing parameters on $E$ or on the target mass number. In this case one should be careful to correctly redefine the dimensions of some arrays like "xcal", "xcen", "ipa", etc., according to the general rules of dynamic dimensioning which have been exposed in Sec.II.1, 
For instance, one can define a new option associated with the parameter "B", which is zero in Tab.IV, by defining " $B=x c a l(11)$ ". In this case, the dimensions of the arrays "xcal" and "ipa" must increase by one unit, because a new independent component of "xcal" has been introduced. The parameter "n10" in Card 1 must increase by one because the total number of "xcal" components directly used in the definition of the OM potential has increased by one. But "m5" of Card 1 must not change. At last, the parameter "ngnpar", belonging the block "common/gdimen/", which specifies the dimensions of "xcal", "xcen", "ipa", etc., as explained in Sec.II.1, must also increase by one unit.

A detailed description of the input of GENOA code can be found in Appendix B.

\begin{tabular}{|c|c|}
\hline \multicolumn{2}{|c|}{ TABLE - IV } \\
\hline "BUFFEG" Parameter & Relation to Central Parameters \\
\hline $\mathrm{RNZ}$ & 1.-2. ${ }^{*} \mathrm{ZT} / \mathrm{AMT}$ \\
\hline $\mathrm{U}$ & $56.3^{*} x \operatorname{cal}(1)-24.0^{*} \mathrm{RNZ}-0.32 * x \operatorname{cal}(2) * \mathrm{E}$ \\
\hline $\mathrm{RR}$ & $1.17^{*} x \operatorname{cal}(3)$ \\
\hline AR & $0.75^{*} x \operatorname{cal}(4)$ \\
\hline WD & $13.0^{*} x \operatorname{cal}(5)-12.0^{*} \mathrm{RNZ}-0.25^{*} \mathrm{xcal}(6) * \mathrm{E}$ \\
\hline WS & $-1.56^{*} \mathrm{xcal}(7)+0.22^{*} \mathrm{xcal}(8)^{*} \mathrm{E}$ \\
\hline RI & $1.26^{*} \mathrm{xcal}(9)$ \\
\hline $\mathrm{AI}$ & $0.58 * x \operatorname{cal}(10)$ \\
\hline USP & $\mathrm{U}$ \\
\hline RS & $\mathrm{RR}$ \\
\hline AS & AR \\
\hline ARE & 0. \\
\hline $\mathrm{ARB}$ & AR \\
\hline $\mathrm{B}$ & 0. \\
\hline WSP & 0. \\
\hline $\mathrm{RC}$ & $R R$ \\
\hline
\end{tabular}




\section{ACKNOWLEDGMENTS}

One of the authors (F. B. G.) would like to express his gratitude for the warm hospitality and the excellent research conditions he found at ORNL. In particular, he is indebted to Dr. Nancy M. Larson for important comments and directives on the structure of SAMMY. F. B. Guimaraes acknowledges the partial support of the Fundação de Amparo à Pesquisa do Estado de São Paulo - FAPESP, Brazil, during the course of this work. 


\section{REFERENCES}

[1] H. Feshbach, A. Kerman and S. Koonin, Ann. Phys. (N.Y.)125, 429 (1980). ; M.B. Chadwick and P.G. Young, Phys. Rev. C47, 2255 (1993).

[2] W. Hauser and H. Feshbach, Phys. Rev.87, 366 (1952).

[3] H. Feshbach, C. E. Porter and V. F. Weisskopf, Phys. Rev.96, 448 (1954).

[4] C. Y. Fu, A Consistent Nuclear Model For Compound and Precompound Reactions with Conservation of Angular Momentum, Technical Report ORNL/TM-7042 (1980), Oak Ridge National Laboratory, U.S.A.

[5] C. Y. Fu, Nucl. Sci. Eng.100, 61 (1988).

K. Shibata and C.Y. Fu Recent Improvements of the TNG Statistical Model Code, ORNL/TM10093, (Aug/1986).

[6] P. E. Hodgson, Nuclear Reactions and Nuclear Structure, (Ed. Clarendon Press, Oxford, England, 1971).

[7] B. V. Carlson, The Optical Model and ECIS95, Tech. Rep. No. H4SMR/921-3 (Ed. International Centre for Theoretical Physics, Trieste, Italy, 1996); Proceedings of the Workshop on Nuclear Reaction Data and Nuclear Reactors, Physics, Design and Safety, Vol.1, pg. 1 (Eds. A. Gandini and G. Reffo, World Scientific, Singapore, 1998).

[8] F. G. Perey, Computer code GENOA - A Searching Procedure for Global Optical Model Parameters, Oak Ridge National Laboratory (1967), U.S.A., unpublished.

[9] D. M. Hetrick, C.Y. Fu and D.C. Larson, Calculated Neutron-Induced Cross Sections for ${ }^{63,65} \mathrm{Cu}$ from 1 to $20 \mathrm{MeV}$ and Comparisons with Experiments, ORNL/TM-9083, ENDF-337 (Aug/1984). 
[10] D. M. Hetrick, D.C. Larson, N.M. Larson, L.C. Leal and S. J. Epperson, Evaluation of ${ }^{28,29,30} \mathrm{Si}$ Neutron Induced Cross Sections for ENDF/B-VI, ORNL/TM-11825, (Apr/1997);

D. M. Hetrick, D.C. Larson, C.Y. Fu, Status of ENDF/B-V Neutron Emission Spectra Induced by 14-MeV Neutrons, ORNL/TM-6637, ENDF-280 (Apr/1979);

D. M. Hetrick, D.C. Larson, C.Y. Fu, Generation of Covariance Files for Isotopes $\mathrm{Cr}, \mathrm{Fe}, \mathrm{Ni}$, $\mathrm{Cu}$, and $\mathrm{Pb}$ in ENDF/B-VI, ORNL/TM-11763, ENDF-350 (Feb/1991).

[11] N.M. Larson, private communication (1999).

[12] N.M. Larson, L.C. Leal and H. Derrien in Efficient Use of Bayes' Equations for Fitting NeutronInduced Cross-Sections and Integral Data, Proceedings of The International Conference on the Physics of Nuclear Science and Technology, Oct/05-08, 1998, New York (Ed. American Nuclear Society, U.S.A., 1998).

[13] L.C. Leal, H. Derrien, N.M. Larson and R. Q. Wright, R-Matrix Analysis of 235 U Neutron Transmission and Cross-Section Measurements in the 0- to 2.25-keV Energy Range, Nucl. Sci. Eng. 131, 230 (1999).

[14] N. M. Larson, Updated Users' Guide for SAMMY: Multilevel R-matrix Fits to Neutron Data Using Bayes' Equations, ORNL/TM-9179, Martin Marietta Energy Systems, Inc., Oak Ridge National Laboratory (August 1984); see also ORNL/TM-9179/R1 (July 85),/R2 (June 89), /R3 (September 96) and /R4 (December 98), Oak Ridge National Laboratory, U.S.A.

[15] N.M. Larson, Introduction to the Theory and Analysis of Resolved (and Unresolved) Neutron Resonances via SAMMY, ORNL/M-6576 - Proceedings of the Workshop on Nuclear Reaction Data and Nuclear Reactors - Physics, Design and Safety - Feb/23 - Mar/27 (1998), I.C.T.P. Trieste, Italy.

[16] P.G. Young, E.D. Arthur and M.B. Chadwick, Comprehensive Nuclear Model Calculations: Introdution to the Theory and Use of the GNASH Code, Technical Report LA-12343-MS (July 1992), Los Alamos National Laboratory, U.S.A.; Proceedings of the Workshop on Nuclear Reaction Data and Nuclear Reactors, Physics, Design and Safety, Vol.1, pg. 227 (Eds. A. Gandini and G. Reffo, World Scientific, Singapore, 1998).

[17] F. G. Perey, Phys. Rev.131, 745 (1963). 
[18] J.J. Griffin, Phys. Rev. Lett.17, 478 (1966).

[19] C. Y. Fu, Nucl. Sci. Eng.86, 344 (1984).

[20] C. Y. Fu, Nucl. Sci. Eng.92, 440 (1986).

[21] B.W. Bush, G.F. Bertsch and B.A. Brown, Phys. Rev. C45, 1709 (1992).

[22] I. Ribansky, P. Oblozinsky, and E. Betak, Nucl. Phys. A205, 545 (1973).

[23] C. Wasshuber PhD. Thesis About Single-Electron Devices and Circuits, Technischen Uni- versit'at Wien Fakult'at f'ur Elektrotechnik, Vienna, January 1997 (e-address: http://www.iue.tuwien.ac.at/diss/wasshuber/diss/node85.html).

[24] C. K. Cline and M. Blann, Nucl. Phys. A172, 225 (1971).

[25] T. Ericson, Adv. in Phys. 9, 423 (1960).

[26] C. Kalbach, Z. Physik A283, 401 (1977).

[27] A. Gilbert and A. G. W. Cameron, Can. J. Phys. 43, 1446 (1965).

[28] A.V. Ignatyuk and Yu. Sokolov, Sov. J. Nucl. Phys, 17, 376 (1973).

[29] C. Y. Fu, Multi-Step Hauser-Feshbach Codes with Precompound Effects: A Brief Review of Current and Required Developments and Applications up to $40 \mathrm{MeW}$, p. 453, Proceedings of the Symposium on Neutron Cross Sections from 10 to $40 \mathrm{MeV}$, Eds. N. R. Bhat and S. Pearlstein, Brookhaven National Laboratory, Upton, New York 11973, May 3-5, 1977, BNL-NCSù50681 (1977).

[30] C. Y. Fu, Atomic Data and Nucl. Data Tables 17, 127 (1976).

[31] C. Y. Fu and F. 13. Perey, J. Nucl. Mat. 61, 153 (1976).

[32] H.A. Bethe, Phys. Rev.50, 332 (1936).

H.A. Bethe, Rev. Mod. Phys.9, 69 (1937).

H.A. Bethe, Phys. Rev.53, 675 (1938).

[33] F. C. Williams, Jr., Nucl. Phys. A166, 231 (1971). 
[34] P.Ring and P.Shuck - The Nuclear Many-Body Problem (Ed. Springer-Verlag, New York, 1980). A.L.Fetter e J.D.Walecka - Quantum Theory of Many-Particle Systems (Ed. McGraw Hill, New York, 1971).

[35] L.N.Cooper, Phys. Rev. 104, 1189 (1956).

[36] J.Bardeen, L.N.Cooper e J.R.Schrieffer, Phys. Rev. 106, 162 (1957);

J.Bardeen, L.N.Cooper e J.R.Schrieffer, Phys. Rev. 108, 1175 (1957).

[37] L.P.Gorkov, Sov. Phys. JETP 34(7), 505 (1958);

N.N.Bogoliubov, Sov. Phys. JETP 34(7), 41 (1958);

N.N.Bogoliubov, Sov. Phys. JETP 34(7), 51 (1958).

[38] L.G. Moretto, Nucl. Phys.A243, 77 (1975).

[39] C. Kalbach, Nucl. Sci. Eng.95, 70 (1987).

[40] W. Dilg, W. Schartl, H. Vonach and M. Uhl, Nucl. Phys.A217, 269 (1973).

[41] S.N. Grimes, R.C. Haight, K.R. Alvar, H.H. Barschall, and R.R. Borchers, Phys. Rev. C19, 2127 (1979);

R.C. Haight and S.N. Grimes, Lawrence Livermore Laboratory Report UCRL-80235 (1977) and private communication.

[42] A. V. Ignatyuk, J. L. Weil, S. Raman and S. Kahane, Phys. Rev. C47, 1504 (1993)

[43] C.R. Wie in Applets for Fermi Distribution

(e-address: http://www.acsu.buffalo.edu/wie/applet/fermi/;

e-address: http://www.acsu.buffalo.edu/ wie/applet/fermi/fermifunction/fermifunction.html).

[44] R. D. Woods and D. S. Saxon, Phys. Rev.95, 577 (1954).

[45] J.E. Lynn, Nuclear Fission and Neutron-Induced Fission Cross-Sections, p.204, Ed. A.Michaudon, Pergamon Press (1981);

X. Bjornholm and J.E. Lynn, Rev. Mod. Phys.52, 1980 (725).

[46] J.H.D. Jensen and J.M. Luttinger, Phys. Rev.86, 907 (1952).

[47] U. Facchini and E. Saetta-Menichella, Energ. Nucl. 15, 54 (1968). 
[48] G. Reffo and M. Herman, Lett. il Nuovo Cim., 34,261 (1982).

[49] I. E. McCarthy, Nuclear Reactions, (Ed. Pergamon Press Ltd., London, 1970).

[50] J. M. Blatt and V. F. Weisskopf, Theoretical Nuclear Physics, (Ed. John Wiley \& Sons, New York, 1952).

[51] Eric W. Weisstein, Newton-Cotes Formulas for Numerical Integration

(Web-Page: Eric Weisstein's World of Mathematics, (C)1996-2000 Eric W. Weisstein and Wolfram Research, Inc.)

[52] J. Raynal, Notes on ECIS94, Note CEA-N-2772, Centre d'Etudes de Saclay (September 1994) 


\section{APPENDIX A - DESCRIPTION OF TNG INPUT (UNIT-60 AND}

\section{UNIT-63)}

We give here a comprehensive description of the input data for TNG (see also Ref. [ $[5]$ ).

The input data are read from unit-60 by the "MAIN" program for the binary reactions and from unit- 63 by the TNG and INPUT subroutines for the tertiary and multistep reactions.

Cross-sections and spectra are given on unit-61 with many printing options. Particle and gamma-ray distributions in ENDF formats are on unit-62. Details of preequilibrium calculation is saved on unit-64.

\section{A.1 INPUT FOR THE BINARY SYSTEMS ON UNIT-60}

Cards 1-3: $3(10 \mathrm{~A} 8) \quad$ Initial comments to characterize the reaction and the theoretical parameters to be used in the data evalution.

Card 4: (11I3,5I5)

$\mathrm{NBS}=$

1

2

3

$\mathrm{NE}=$

ISTEP1 $=$

INGMN $=$

NǴSP $=$

INGMX =

IPRINT $=$

0

1

IPCOEF =

0

1
Number of binary systems

$(\mathrm{n}, \mathrm{n})$

$(\mathrm{n}, \mathrm{n})(\mathrm{n}, \mathrm{p})$

$(\mathrm{n}, \mathrm{n})(\mathrm{n}, \mathrm{p})(\mathrm{n}, \mathrm{a})$

Number of incident energies

Number of integration steps for width fluctuation integrals $(>20)$

Minimum angle of angular distributions in deg (normally 0 )

Angular spacing in deg (at least $5 \mathrm{deg}$ )

Maximum angle in deg (normally 180)

Printing control

Minimum output on unit 61

24 options controlled by Card 6

Printing control

No print

Print Legendre coefficients for discrete levels in ENDF/B format on unit 62 


\begin{tabular}{|c|c|}
\hline IFLC $=$ & Width fluctuation control \\
\hline 0 & Do not want it \\
\hline 1 & Want it (discrete levels and continuum) \\
\hline $\mathrm{IPCH} 91=$ & Printing control \\
\hline 0 & No print \\
\hline 1 & Print $\mathrm{ENDF} / \mathrm{B} \mathrm{MF}=5, \mathrm{MT}=91$ on unit 62 if ITNG $=0$ (see Card 15 below) \\
\hline ICOMEL $=$ & Printing control \\
\hline 0 & No print \\
\hline 1 & $\begin{array}{l}\text { Print compound elastic scattering cross-sections and Legendre coefficients on unit } \\
62 \text { for use in optical-model GENOA }\end{array}$ \\
\hline MAT $=$ & ENDF/B material number \\
\hline $\mathrm{MT}(\mathrm{I})(\mathrm{I}=1, \mathrm{NBS})=$ & ENDF/B reaction number for the I-th reaction \\
\hline
\end{tabular}

Card 5: (2013)

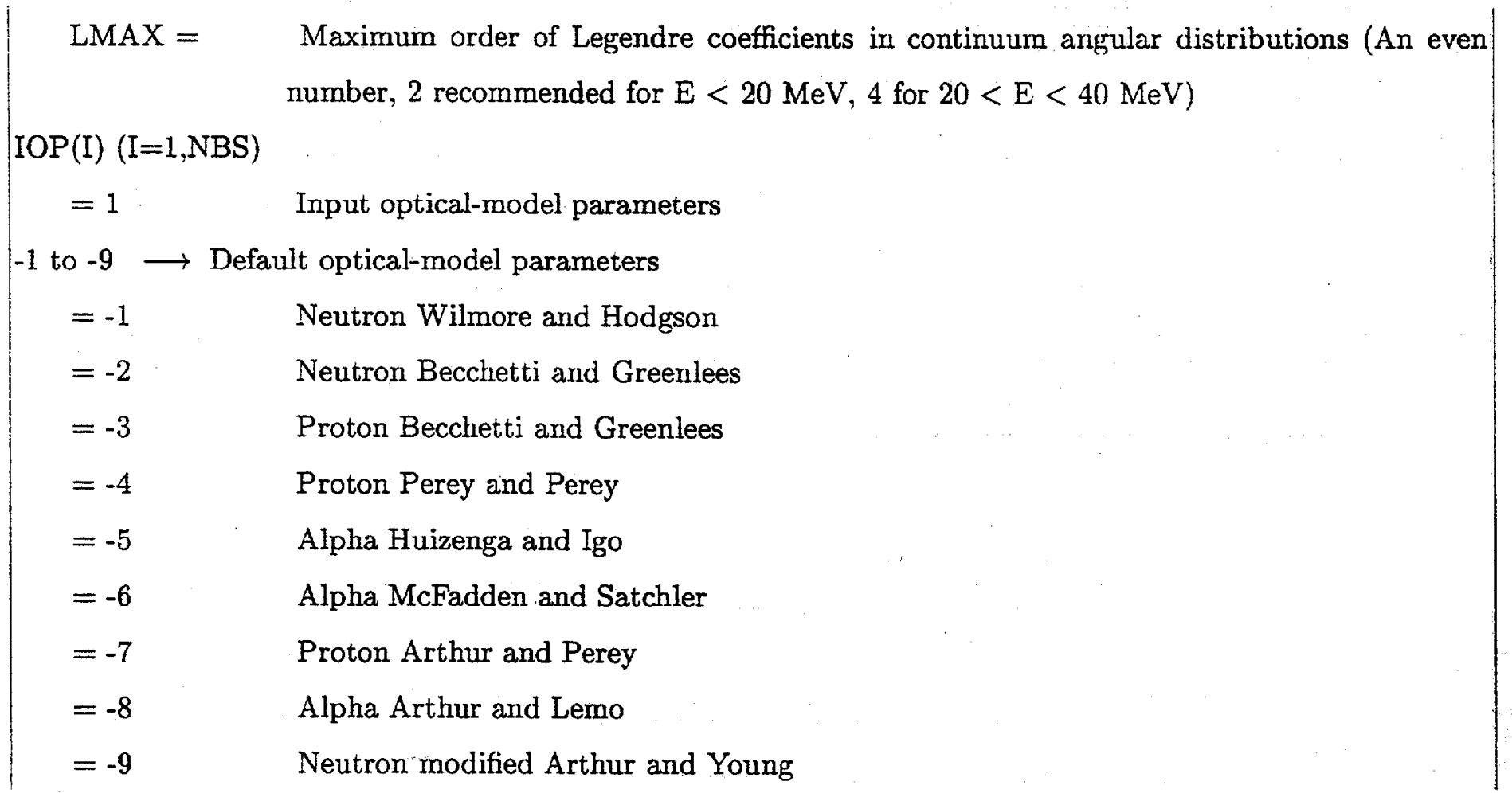

See details in subroutine "globe", source file "tng1.f". 
Card 6: (80I1) Skip if IPRINT $=0$

$\operatorname{IPRT}(\mathrm{I}),(\mathrm{I}=1.80)$
$=1$
Print specified quantities listed in last part of Appendix A (section A.3 )
$=0$
Do not print

Card 7: (80I1) Skip if LMAX =0

IANGL(I) = Continuurn angular distribution control for $(I=1,80)$ the $I$-th bin
0
No angular distribution
1 Angular distribution for compound reaction only
2
Augular distribution for both compound and precompound reactions
Continuum bins for different reactions are stacked. The number of bins with IANGL =
1 or 2 should be less than 10 .

Card 8: (8E10.3).

$\mathrm{BN}=\quad$ Binding energy $(\mathrm{MeV})$ of incident particle in composite system

$\mathrm{RAD}=\quad$ Radiative capture control

$0.0 \quad$ No capture

1.0 Want capture (more input in unit 63 with NTR = 1; see below)

FISS $=\quad$ Fission control

$0.0 \quad$ No fission

$1.0 \quad(\mathrm{n}, \mathrm{f})$

$2.0 \quad(n, f)(n, n f)$

(n.f) (n,nf) (n,2nf)

and so on

ECENDF $=\quad$ not used. See input for unit-63, Card 10 (section A.2)

CCN3 $=\quad$ correlation coefficient, for $\mathrm{n}=3$, for precompoud angular distribution (normally 0.5 )

OMDI $=\quad$ a constant level density added to the $n=2$ part of the residual level density in $\left(n, n^{\prime}\right)$,

to alter the precornpound spectral shape (needed only for spherical nuclei such as $\mathrm{Pb}$ and rotational ones such as $\mathrm{W}$ to simulate collective effects in the continuum) 
Card s 9-13: Repeated NBS times (NBS = Number of binary systerns -Card 4)

Card 9: $(6 \mathrm{~F} 7.3, \mathrm{~A} 8)$
$\mathrm{AT}=$
$A$ of residual nucleus
$\mathrm{ZT}=$
$\mathrm{Z}$ of residual nucleus
$\mathrm{AI}=$
A of outgoing particle
$\mathrm{ZI}=$
$\mathrm{Z}$ of outgoing particle
$\mathrm{Q} 0=$
$\mathrm{Q}$-value for reaction in $\mathrm{MeV}$
$\mathrm{AII}=$
Spin of outgoing particle
HID $=$
Reaction identification such as $(n, p)$

(use $A I=1$ for neutron and proton, 4 for alpha, 0 for photon)

Card 10: (2013)

NLVL1 $=\quad$ Number of discrete levels in residual nucleus (at least 2 and at most 40)

Card 11: (5(F5.3,F4.1,F4.0,I3)) - Parameters describing the discrete levels of the residual nucleus $\mathrm{ENU}=\quad$ Excitation energy of a discrete level in $\mathrm{MeV}$

$\mathrm{AIT}=\quad$ Spin of a discrete level

$\mathrm{AP}=\quad$ Parity of a discrete level

$-1.0 \quad$ Negative

$+1.0 \quad$ Positive

IDOUT $=\quad$ Printing control

$1 . \quad$ Nothing printed

2 Integrated cross sections in $\mathrm{mb}$

3 Angular differential cross sections in $\mathrm{mb} / \mathrm{sr}$

There can be 120 levels in all 3 binary systems, but at most 40 of them can have IDOUT $=3$.

If none of IDOUT is 3 and LMAX $=0$, all angular dependent calculations are skipped. Code allows. overlapping of discrete levels and continuum. 
Card 12: (7f7.3,i3,3f7.3,i3)

Gilbert-Cameron composite level density parameters

$\mathrm{EC}=\quad$ Energy of continuum cut-off (may be less than some of the ENU's) in $\mathrm{MeV}$

$\mathrm{EX}=\quad$ Energy of the tangency point in $\mathrm{MeV}$

$\mathrm{E} 0=\quad$ Energy shift in $\mathrm{MeV}$ in the constant-temperature formula

$\mathrm{TM}=\quad$ Constant temperature "T" in $\mathrm{MeV}$

$\mathrm{AC}=\quad$ The Fermi gas parameter, " $a "$, in $1 / \mathrm{MeV}$

$\mathrm{CC}=\quad$ The spin cut-off parameter, $\sigma(U)$, is given by

$$
\begin{aligned}
\sigma(U) & =\left(\frac{6}{\pi^{2}}\right) c_{\sigma} A^{2 / 3}(a U)^{1 / 2} \\
& =c_{\sigma} A^{2 / 3}\left(\left(\frac{6}{\pi^{2}}\right) g U\right)^{1 / 2}
\end{aligned}
$$

where the relation $(\underline{3.26})$ has been used and "c $c_{\sigma}$ " is the parameter "CCSIG" in Card 14 below.

DELT $=\quad$ Odd-even (pairing) correction " $\mathrm{U} "$ in $\mathrm{MeV}$

ICOUT $=\quad$ Printing cotrol, similar to IDOUT (If ICOUT $=3$, must also have LMAX $i 0$ )

$1 \quad$ Nothing printed

2 Integrated cross sections in mb

3 - Differential cross sections in $\mathrm{mb} / \mathrm{sI}$

Normally " $\sigma(U)$ " (parameter "CC" above) varies between $0.0888 a A^{2 / 3}$ (Jensen and Luttinger [46]) and $0.146 a A^{2 / 3}$ (Facchini and Saetta-Menichella [47]]).

If $\mathrm{EX}=\mathrm{EC}$, only the Fermi-gas part of the composite formula is used.

If $\mathrm{EX}>99$, only the constant-ternperature part is used. TM is input and EO is automaticcally adjusted to the number of discrete levels below EC.

If $T M=0$ but $E X$ is not equal to EC, the code will search for EX, E0, and TM, starting from the other parameters and the given number of discrete levels below EC (the discrete levels below EC is supposedly complete). 
Card 13: (8F7.3,2I3/8F7.3) Present if $\mathrm{IOP}(\mathrm{I})=1$.

Optical-model parameters

$\begin{array}{ll}\mathrm{U}= & \text { Real well depth in MeV } \\ \mathrm{UE}= & \text { E-dependence of real potential } \\ \mathrm{RU}= & \text { Radius of real potential in fm } \\ \mathrm{AU}= & \text { Diffuseness of real potential in fm } \\ \mathrm{W}= & \text { Imaginary well depth (surface, volume or Gaussian depending on IFS below) } \\ \mathrm{WE}= & \text { E-dependence of W } \\ \mathrm{RW}= & \text { Radius of imaginary potential in fm } \\ \mathrm{AW}= & \text { Diffuseness of imaginary potential in fm } \\ \mathrm{IFS}= & \text { Index for potential form-factor } \\ 1 & \text { Woods-Saxon surface } \\ 0 & \text { Woods-Saxon volume } \\ -1 & \text { Gauss } \\ \mathrm{ISTEP}= & \text { Number of steps used in optical-model calculations (40) } \\ \text { WV }= & \text { Volume part of imaginary well depth in MeV (additional to W if IFS = 1) } \\ \text { WVE }= & \text { E-dependence of WV } \\ \mathrm{UEE}= & \text { E**2 dependence of real potential in } 1 / \mathrm{MeV} \\ \mathrm{RC}= & \text { Coulomb radius in fm (The real potential is assumed to have Woods-Saxon form factor. } \\ & \text { E is the laboratory energy.) }\end{array}$


Card 14: (2F7.0,E7.1,6F7.0)

$\mathrm{ACC}=\quad$ Level density parameter ' $a$ ' for composite system in $1 / \mathrm{MeV}$

$\mathrm{QC}=$ Pairing correction for composite system in $\mathrm{MeV}$ (used to calculate $\Delta_{0}$ in subroutine "pair1", in the source file "tng1.f").

"QC" can be negative if the back-shifted fermi gas model is used.

In this case the spin cut-off function is given by the expression of Reffo and Herman, Ref. $[\underline{48}]$,

$$
\sigma^{2}(U, n)=0.28 \tilde{n} A^{2 / 3}
$$

where $\tilde{\pi}$ is the most probable exciton number, which is a function of " $U$ ".

TCUT $=\quad$ Transmission coefficient cut-off (1.0E-3 for test calculation, 1.0E-7 for production runs)

$\mathrm{FA}=\quad$ Clustering probability for alpha $(0.1-0.2)$

$\mathrm{FG}=\quad$ Scale factor for precompound mode of (n,gamma) reaction (1.0)

$\mathrm{F} 2=\quad$ Scale factor for mean-square matrix element $\mathrm{MeV}^{* *} 3(4.0-7.0)$

$\mathrm{TIME}=\quad$ cutoff time in arbitrary units set internally for integration of master equation (1000.0)

$\mathrm{FP}=\quad$ Scale factor for precompound mode of $(n, p)$ reaction (1.0)

CCSIG $=\quad$ Constant factor in the evaluation of the mean-square of magnetic quantum number for single particle states

$$
<m^{2}>=0.146 A^{2 / 3} \text { (Jensen and Luttinger) }
$$

or

$$
<m^{2}>=0.240 A^{2 / 3} \text { (Facchini and Saetta-Menichella) }
$$

$<m^{* * 2}>=$ CCSIG*A** $^{*}(2 / 3)$, with "CCSIG" normally varying between 0.146 [느] and $0.240[\underline{47}]$

Repeat Cards 15-19 NE times, each for one energy. 
Card 15: (3I5,7E10.0).

ISDI $\quad=0 \quad$ No direct interaction cross-section input

$=1$ Direct-interaction cross-section input for discrete levels (needed only for gamma-ray production calculations).

If ISDI $=1$, effects of direct interaction are included automatically and should not be included again in OTHER below.

ITNG $\quad=0 \quad$ Single step (binary) calculation

$=1 \quad$ Multistep calculations

The capture process $(\mathrm{RAD}=1)$ is single-step, even though gamma-ray branching ratios for discrete levels of the compound nucleus are input in unit-63.

IPRE $\quad=0 \quad$ Hauser-Feshbach only

$=1$ Hauser-Feshbach and Precompound

$=2$ Precompound only (for odd-A target)

There must be some states excited into the continuum to calculate the precompound cross sections, so make sure that " $E$ " is somewhat greater than all the "EC"'s to use IPRE = 1 or 2.

$\mathrm{E} \quad=\quad$ Incident particle energy, laboratory $(\mathrm{MeV})$

DBIN $\quad=\quad$ Bin width $(\mathrm{MeV})$ for outgoing particle energies and continuum, must be such that the number of bins per nucleus is at most 40 . If negative, variable bin widths are used (additional input on Cards 16 - 17). If zero, the code uses the same bins as in the previous energy run.

EGBIN = Bin width for emitted gamma-ray energies in $\mathrm{MeV}$ (1200 bins allowed, code will thin results to keep narrow bins for large discrete gamma-rays)

OTHER $=$ Cross section in mb for reactions not included in the calculation for normalization.

Cards 16-17: 2(20F4.1) Skip if DBIN is not negative.

$\operatorname{BINI}(I)(I=1,40)=$

Variable bin widths in $\mathrm{MeV}$ 
Cards 18-19: Repeated NBS times if ISDI $=1$

Card 18: (I5)

NSDI $=$ Number of levels for which direct-interaction cross sections are to be put in for the I-th residual nucleus

Card 19: (15,E10.4)

There are NSDI pairs of NXW and SDI.

NXW $=\quad$ Level number of current residual nucleus (NXW $=1$ for ground state)

$\mathrm{SDI}=\quad$ Direct-interaction cross section in $\mathrm{mb}$ for the NXW-th level

\section{A.2 INPUT FOR TERTIARY AND MULTISTEP REACTIONS ON UNIT-63}

The following input is specific for subroutines "INPUT" and "TNG", in the file "/tng/tnga.f".

Cards 1-17: Repeat NBS times if both RAD and FISS are equal to zero.

Repeat (NBS + 1) times if either RAD or FISS is not equal to zero.

Card 1: (16I5)

MAT $=$

NTR $=$

IPCHN = 0

1

$\mathrm{IPCHG}=$ 0 1
ENDF/B material number

Nurnber of tertiary reactions. Capture and Fission reactions should not be counted in NTR.

Printing control for particle production spectra in ENDF formats on Unit 62

Do not print

Print

Printing control for gamma-ray production spectra in ENDF formats on Unit 62

Do not print

Print (see also IPRT(18) in Card 6 of Unit 60 for printing gamma-ray production spectrum for each reaction on Unit 61) 
Card 2: $(8 \mathrm{~F} 10.0)$

EMRAT $=\quad$ Fraction of $\mathrm{M} 1$ strength at $7 \mathrm{MeV}$ relative to $\mathrm{E} 1$ (0.1 recominended, 0.1 means $10 \% \mathrm{M} 1$ and $90 \% \mathrm{E} 1$ )

EERAT $=\quad \mathrm{E} 2 / \mathrm{E} 1$ and M2/M1 assuming same energy dependence for continuum-to discrete transitions only (0.01 recommended)

Cards 3-11: Repeated NTR times

Card 3: (A4)

TITLE $=\quad$ Title, such as n,np Gamina-ray channels such as (n,ng) inust come first.

Card 4: (16I5)

$\mathrm{NLEV}=\quad$ Number of discrete levels of residual nucleus for the I-th reaction (at least 2, at most 40)

NTPCRD $=$ Number of Cards containing gamma-ray branching ratios (at least 1 if gamma production wanted)

0 if gamma-ray production not wanted

One should be careful not to double count the gamma-rays from a given reaction. For example, the gamma-ray production from $(n, 2 n)$ can be included in either the 2nd step or the 3rd step but should not be in both.

$\mathrm{N1}=\quad$ Index for tallying particle $\mathrm{y}$ in $(\mathrm{x}, \mathrm{yz})$ reaction for total particle production cross sections $0 \quad$ Do not tally

1 Tally it as a neutron

$2 \quad$ Tally it as a proton

$3 \quad$ Tally it as an alpha

$\mathrm{N} 2=\quad$ Index for tallying particle $\mathrm{z}$ in $(\mathrm{x}, \mathrm{yz})$ reaction for total particle production cross sections, same conventions as for $\mathrm{N} 1$

$\mathrm{MT}=\quad$ Reaction number in $\mathrm{ENDF} / \mathrm{B}$ format 
NNUC $=1 \quad$ Input optical-model parameters

-1 to $-9 \longrightarrow$ Default optical-model pararneters

$$
\begin{aligned}
& =-1 \quad \text { Neutron Wilmore and Hodgson } \\
& =-2 \quad \text { Neuttron Becchetti and Greenlees } \\
& =-3 \quad \text { Proton Becchetti and Greenlees } \\
& =-4 \text { Proton Perey and Perey } \\
& =-5 \quad \text { Alpha Huizenga and Igo } \\
& =-6 \quad \text { Alpha McFadden and Satchler } \\
& =-7 \quad \text { Proton Arthur and Perey } \\
& =-8 \quad \text { Alpha Arthur and Lemo } \\
& =-9 \quad \text { Neutron modified Arthur and Young } \\
& \text { NGAM }=1 \text { giant dipole parameters read in Card } 9 \\
& =0 \quad \text { Use defaults }
\end{aligned}
$$

NCAP $=\quad$ Number of Cards containing primary s-wave capture gamma-ray branching ratios read in

\section{Card 11}

$0 \quad$ The code calculates the branching ratios

NCAP is needed only for $(n, \gamma)$ reaction. Otherwise, it should be set to 0 .

Card 5: (8F10.0)
$\mathrm{AT} 1=$
Mass of residual nucleus
$\mathrm{AII}=$
Mass of outgoing particle
$\mathrm{ZT} 1=$
Charge of residual nucleus
$\mathrm{ZI} 1=$
Charge of outgoing particle
$\mathrm{SI} 1=$
Spin of outgoing particle ( 0.0 for gamma-rays)
$\mathrm{Q} 2=$
Q-value in $\mathrm{MeV}$

Card 6: (5(F5.3,F4.1,F4.0,3X))

There are NLEV discrete levels.

ELEV $=\quad$ Excitation energy $(\mathrm{MeV})$

SPIN $=\quad$ Spin

PAR $=\quad$ Parity 
Card 7: $(8(2 \mathrm{I} 3, \mathrm{~F} 4.2))$ Skip if NTPCRD $=0$

$\mathrm{L}=\quad$ - Index for initial level of gamma-ray transition

$M=\quad$ Level number for final level (1 for ground state)

$\mathrm{BR}=\quad$ Branching ratio for transition from $\mathrm{L}$ to $\mathrm{M}$

$2.0 \quad 0+$ to $0+$ transition $(0.51-\mathrm{MeV}$ gamma rays)

3.0 Isomeric state $L$ (use $M=L-1$ )

The sets of $L, M, B R$ for $B R=0$ need not be put in. Unless $B R=2$. or 3., sum of $B R$ over $M$ should be 1 .

Card 8: (8F7.0,2I3/8F7.0).

Present if NNUC $=1$, skip if $(n, g)$, (n,ng), etc, for which NNUC $=0$.
$\mathrm{U}=$
Real well depth in $\mathrm{MeV}$
$\mathrm{UE}=$
E-dependence of real potential
$\mathrm{RU}=$
Radius of real potential in $\mathrm{fm}$
$\mathrm{AU}=$
Diffuseness of real potential in $\mathrm{fm}$
$\mathrm{W}=$
Imaginary well depth in $\mathrm{MeV}$ (surface, volume or Gaussian depending on IFS below)
$\mathrm{WE}=$
E-dependence of imaginary potential
$\mathrm{RW}=$
Radius of imaginary potential in fm
$\mathrm{AW}=$
Diffuseness of imaginary potential in $\mathrm{fm}$
IFS $=1$
Woods-Saxon surface
0 Woods-Saxon volume
$-1 \quad$ Gauss
ISTEP $=\quad$ Number of steps used in optical-model calculations (40)
$\mathrm{WV}=\quad$ Volume part of imaginary depth in $\mathrm{MeV}$ (additional to $\mathrm{W}$ if IFS $=1$ )
WVE $=\quad$ E-dependence of WV
$\mathrm{UEE}=\quad \mathrm{E}^{* *} 2$-dependence of real potential in $1 / \mathrm{MeV}$
$\mathrm{RC}=\quad$ Coulomb radius in fro

The real potential is assurned to have Woods-Saxon form factor. E is the laboratory energy. 
Card 9: (8F10.0) Present if NGAM = 1. - Giant dipole resonance parameters (Lorentzian form)

$\mathrm{E} 1=\quad$ Resonance energy in $\mathrm{MeV}$

GAMI $=\quad$ Full width at half maximum in $\mathrm{MeV}$

SIG1 $=\quad$ Peak cross section in mb

GSTEP $=\quad$ Energy below which GDR cross section is multiplied by GFRAC

GFRAC $=\quad$ Factor referred to above

$\mathrm{E} 2=\quad$ Energy for the second resonance in $\mathrm{MeV}(=0$ if single peak)

GAM2 $=\quad$ Full width at half maximum for the second resonance

SIG2 $=\quad$ Peak cross section for the second resonance

Card 10: $(11 \mathrm{f7} .0, \mathrm{i} 3)$

Level density parameters
$\mathrm{EC}=\quad$ Energy for the continuum cut-off in $\mathrm{MeV}$
$\mathrm{EX}=\quad$ Energy of the tangency point in $\mathrm{MeV}$
$\mathrm{E} 0=\quad$ Energy shift in constant-temperature formula in $\mathrm{MeV}$
$\mathrm{TM}=\quad$ Constant temperature "T" in $\mathrm{MeV}$
$\mathrm{AC}=\quad$ The Fermi gas parameter, "a" in $1 / \mathrm{MeV}$
$\mathrm{CC}=\quad$ Spin cut-off factor "c"
DELC $=\quad$ Odd-even pairing correction " $\mathrm{U}$ " in $\mathrm{MeV}$
ECENDF $=$ Continuum cutoff in $\mathrm{MeV}$ used in ENDF/B-VI File 6, MT = 91, 649, or 869.

ECENDF is normally equal to EC, the continuum cut-off defined in Card 10, but may be set less than $\mathrm{EC}$ to include some high-lying levels in the ENDF/B continuum.

If $E C E N D F=0$, all discrete cross sections are included in the ENDF/B continuurn, as the case for $\mathrm{MT}=16,22$ and 28 for which there are no ENDF MT values assigned to the discrete cross sections.

Card 11: (10(13,F5.0)) Skip if NCAP $=0$

There rnust be NCAP Cards.
$\mathrm{MC}=$
Level number for final state
$\mathrm{BRC}=$
Branching ratio for primary transition to $\mathrm{MC}$ 
If the sum of BRC over MC is less than 1.0, the code combines the input BRC's with calculated ones. If NTPCRD $>0$ but NCAP $=0$, calculated primary gamma-ray branching ratios will be used.

Cards 12-16: Present if fission cross sections is wanted in this step.

Card 12: $(A 4,15)$

TITLF $=\quad$ Reaction identification such as (n,nf)

$\mathrm{MTF}=\quad$ Reaction number in $\mathrm{ENDF} / \mathrm{B}$ format

Card 13: (8F10.0)

Parameters for the double-humped barrier model

$\mathrm{EB}(1)=\quad$ Inner barrier height in $\mathrm{MeV}$

$\mathrm{EB}(2)=\quad$ Outer barrier height in $\mathrm{MeV}$

$\mathrm{HOM}(1)=\quad$ Inner barrier curvature in $\mathrm{MeV}$

$\operatorname{HOM}(2)=\quad$ Outer barrier curvature in $\mathrm{MeV}$

If $\mathrm{EB}(2)=0.0$, the single-humped barrier model is employed.

Cards 14-16: Repeated twice if the double-humped barrier model adopted.

Card 14: (16I5)

NLEVF $=\quad$ Number of discrete levels at a barrier

Card 15: (5(F5.3,F4.1,F4.0,3X))

ELEVF $=\quad$ Excitation energy in $\mathrm{MeV}$ relative to the barrier.

SPINF $=\quad$ Spin of the level

PARF $=\quad$ Parity of the level

$-1.0 \quad$ Negative

$+1.0 \quad$ Positive 
Card 16: (8F7.0)

Level density parameters for transition states

$\begin{array}{ll}\mathrm{ECF}= & \text { Continuum cut-off in } \mathrm{MeV} \\ \mathrm{EXF}= & \text { Tangency point in } \mathrm{MeV} \\ \mathrm{E} 0 \mathrm{~F}= & \text { Energy shift in constant-temperature formula in } \mathrm{MeV} \\ \mathrm{TMF}= & \text { Constant ternperature "T" in } \mathrm{MeV} \\ \mathrm{ACF}= & \text { "a" in units of } \mathrm{MeV}-1 \\ \mathrm{CCF}= & \text { Spin cut-off factor "c" } \\ \mathrm{DELCF}= & \text { Pairing correction " } \mathrm{U} " \text { in } \mathrm{MeV}\end{array}$

NOTE: If TMF and ACF are equal to 0.0, default level density recommended by Lynn is used. The corresponding level density parameters are defined in the subroutine "1vlynn", in the file "/tng/tngb.f", using the level density parameters recommended by J.E.Lynn. [45]

Card 17: (5I5) Skip if $(\mathrm{n}, \mathrm{g})$ or $(\mathrm{n}, \mathrm{f})$ or ITNG $=0$

IA $=$ Reaction index in the previous step from which to branch into current step

$1 \quad(n, n)$

$2 \quad(n, p)$

$3 \quad(n, a)$

IMORE $=0$ Return to previous step

$=1$ More reaction to be done in current step or, if ISTORE $=1$, go to next step

ISTORE $=0$ Do not store current output for next step

$=1$ Store current output

\section{A.3 DETAILS OF CARD 6 ON UNIT-60}

The entries in Card 6 are,

$\operatorname{IPRT}(\mathrm{I}),(\mathrm{I}=1,80)$

$=1 \quad$ Print the specified quantities on unit- 61

$=0 \quad$ Do not print 
The following printed outputs are helpful for understanding the physics in the code. For new users, try one at a time because some of the outputs may be interwoven and hard to read.

$I=1: \quad$ Transmission coefficients for incident neutron and outgoing particles exciting the continuum bins in TRANS subroutine.

2: Giant dipole cross section in INPUT

3: Level density integrated over each bin in INPUT

4: Gamma-ray transmission coefficients in capture in COMPET

5: Gamma-ray transmission coefficients, radiative widths, and width-fluctuation corrections for capture and continum in HOWZT

6: Iteration, in DNCONV, for conversion to single-fermion level density parameters

7: Details of pairing correction in PAIR

8: $\quad$ Spin distribution in formation and $2 \mathrm{p}-1 \mathrm{~h}$ states in DNTRN

9: Inverse reaction cross sections in PRECOA

10: Transmission coefficients in tertiary and higher steps in TRANS1

11: Continuum spin and parity distributions in tertiary and higher steps in NORM

12: Partial gamma-ray production from each spin and parity of continuum states if $>10 \mathrm{mb}$ in CASCAD

13: Spin weights (SWT) of levels in 2 nd and 3rd steps if $\mathrm{IC}=1$, i.e., highest bin in (n,ng) in COMPET

14: Legendre coefficients for each spin and parity of continuum bins with IANGL $=2$ in HOWZT

15: Details in angular momentum coupling in HOWZT

16: Details of precompound calculations in PRECOA and OMEFF in unit 64.

17: Total gamma-ray production spectra in unit 59 in a format readable by GPLT, a code that plot ORELA data and calculated results.

18: $\quad \gamma$-ray production cross sections and energy distributions for individual reactions in CASCAD.

19: First-chance fission transmission coefficients in INPUT

20: Continuum transmission coefficients for fission in TRFISS

21: Primary gamma-ray spectrum from precompound capture in PREG 
22: Cross sections of all discrete gamma rays in CASCAD (cross sections of discrete gamma rays greater than $50 \mathrm{mb}$ are always printed)

23: Competition of tertiary reactions within each binary bin in NNN.

24: Total gamma-ray production spectra in TNG (see 17 for printing on unit 59).

25: Details in PAIR2 (two-Fermion p-h level densities for tng2 only, not used for tng1).

80: Used internally for printing input data for the first incident energy only. 


\section{APPENDIX B - DESCRIPTION OF GENOA INPUT (UNIT-50)}

We give here a comprehensive description of the input data for GENOA (see also Ref. [8]).

\section{B.1 CARDS COMMON TO ALL DATA SETS}

Card 1: General run parameters. Free format

fadc, fadc1, nadc, icrun, krcan, n10 and m5

fadc $=$

fadcl $=$

nadc $=$

n10

m5

$\mathrm{krcan}=$

Multiplicative factor to be used in "BUFFEG" when "NCOMP" (2nd. entry of Card 7 below) is equal to 4 or 5

Multiplicative factor to be used in "BUFFEG" when NCOMP $=6$

Number of reactions for which the parameter "ADC" in subroutine "BUFFEG" will vary independently. The first "nadc" reactions listed in unit-50 have independent variation of "ADC", each one proportional to a different component of the vector "xcal". The remaining "kto-nadc" reactions (see "kto" in Card 2 below) have the corresponding "ADC" parameters varying proportionally to the same component of "xcal" and, therefore, they are proportional to each other.

icrun $=\quad$ Maximum number of complete sequential runs of the coupled codes GENOA-TNG.

Simultaneous value of "NCOMP" to be used in all reactions after the first run of the coupled codes GENOA-TNG. If "krcan" = -1, the values of "NCOMP" are not altered with respect to the first run.

Number of central parameters directly used in the definition of the O.M. Potential in subroutine "BUFFEG" (the default value of " $n 10$ " is 10). A variation of the value of "n10" requires a corresponding redefinition of "BUFFEG". If nothing is done in "BUFFEG" "n10" must be 10 .

Number of central parameters in "BUFFEG" associated with the definition of the options "NCOMP $=2$ " and "NCOMP = 7 " (the default value of "m5" is 4 , corresponding to 4 independdent components of "xcal" which are variable in the definition of the options "NCOMP $=2 "$ and "NCOMP $=7 "$ ). Other value of "m5" requires a redefinition of "BUFFEG". If no alteration is performed "m5" must be 4 . 
Card 2: Search control Card or Cards. FORMAT(I2,3I1,13,I3,I2/14015)

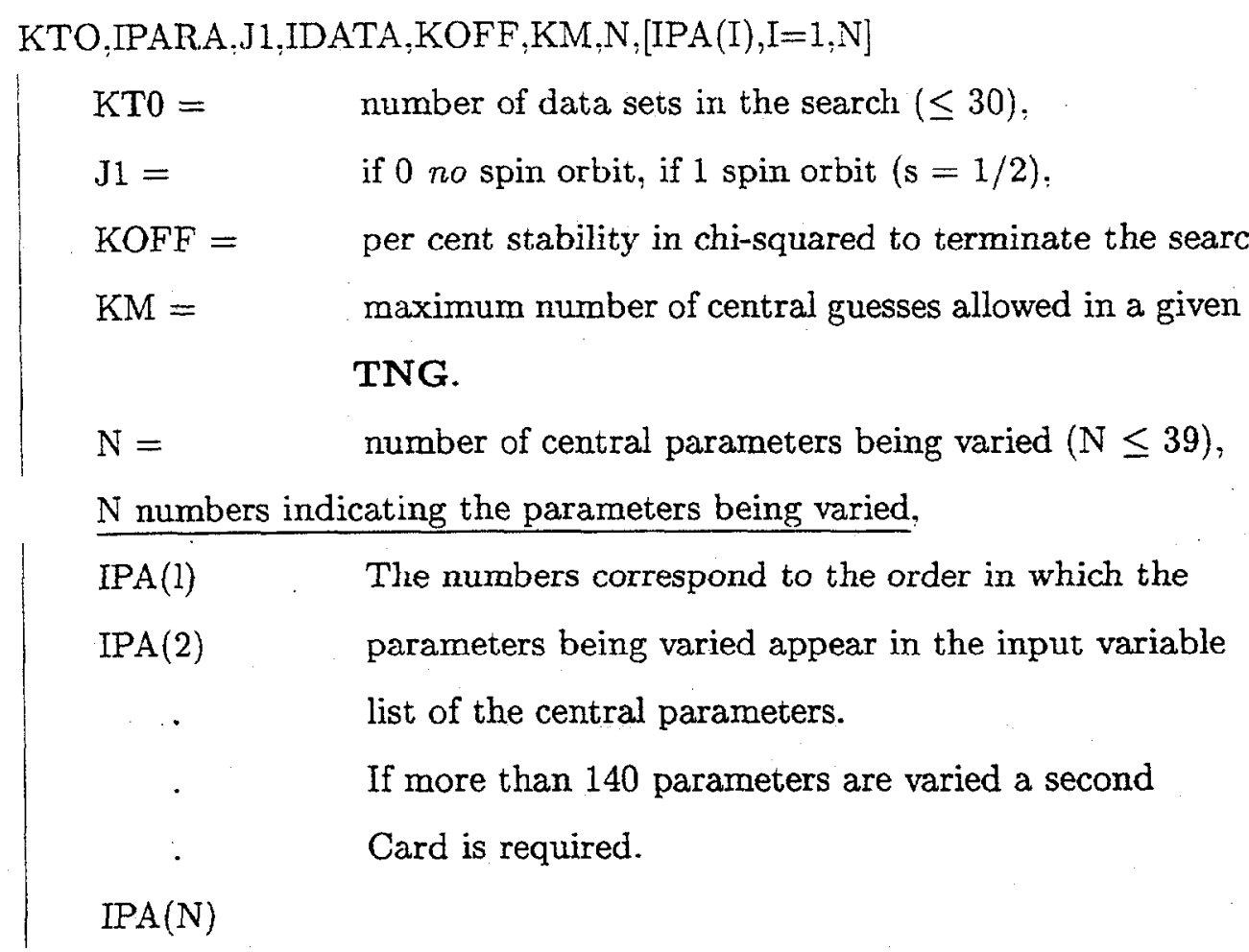

Card 3: Starting value of central parameters (XCAL). Format 8F10.6.

Use as many Cards as necessary to give initial values to the parameters indicated by "IPA".The order on the Card corresponds to the IPA list.

Card 4: Variation Card or Cards. Format 8F10.6

The number of Cards depend on N. A zero in the field will set variation $=10 \%$; otherwise set your own variations. The order on the Card corresponds to the IPA list. 


\section{B.2 SPECIFIC DEFINITIONS FOR EACH EXPERIMENTAL DATA SET}

Card 5: Experimental information. Format (f10.6,4f10.3)

$\begin{array}{ll}\mathrm{E}= & \text { lab energy in Mev } \\ \mathrm{AMT}= & \text { target mass in AMU } \\ \mathrm{AMI}= & \text { incident particle mass in AMU } \\ \mathrm{ZT}= & \mathrm{Z} \text { of target } \\ \mathrm{ZI}= & \mathrm{Z} \text { of incident particle }\end{array}$

Card 6: Numeric information: theoretical angles and normalization parameters.

To be used at the end of the search in the printout of results for this data set, at equally spaced angles. Format $7 \mathrm{f10.3}$

$$
\begin{array}{ll}
\mathrm{H}= & \text { integration step length in fm. } \\
\mathrm{A} 1= & \theta_{\min } \\
\mathrm{A} 2= & \Delta \theta \\
\mathrm{A} 3= & \theta_{\max } \\
& \theta_{\min } \neq 0 \text { for charged particles. }
\end{array}
$$

Up to 200 equally spaced angles can be output.

ANOR $=\quad$ normalization of $\sigma(\theta)$ 's only (1.00 means OK 1.05 means increase $5 \%$, etc.)

OWES $=\quad$ overall weight of $\sigma(\theta)$ 's this has most meaning with

OWEP $=\quad$ overall weight of $P(\theta)$ 's respect to other data sets.

The weight on each $\sigma(\theta)$ point is

$$
\frac{\text { OWES }}{\Delta \sigma(\theta)} \longleftarrow \text { absolute }(\mathrm{mb} / \mathrm{str})
$$

The weight on each $P(\theta)$ point is

$$
\frac{\text { OWEP }}{\Delta P(\theta)} \longleftarrow \text { absolute }
$$

GENOA minimizes the sum of all chi squares for all distributions. If one desires to weigh the distributions such that the code minimizes the chi square per point, the weight to use for each data set is $1 / \sqrt{n}$, where $\mathrm{n}$ is the number of data points in each data set. 
Card 7: Options for Cross-Section evaluation. Format (i2,4i1,2i2,f10.3)

LMX, NCOMP, NR, IPLT, NT, NS, NP and THEP.

LMX $=$ Maximum number of partial waves for this data set

NCOMP $=$ The code finds the magnitude of the compound elastic contribution in accordance with the various options associated with "NCOMP".

NCOMP $=\quad 1 \quad$ ADC varies independently for each reaction with values close to 1.

NCOMP $=2$ In this option the energy-dependent magnitude of the compound elastic contribution is represented by the empirical relation,

$$
\sigma_{C E}(E)=A /[1+\exp [(B-E) / C]]
$$

where each one of the parameters "A", "B" and "C", vary independently.

NCOMP $=3 \quad$ ADC has fixed value equal to zero.

$4 \quad \mathrm{ADC}$ has fixed value equal "fadc" (taking "fadc" $=1$ one recovers the original "NCOMP=1" definition).

$5 \quad \mathrm{ADC}$ varies independently for each reaction with values close to "fadc".

6 ADC varies independently for each reaction with values close to "fadcl". Usually "fadc1" is different of "fadc" to set different rates of convergence for the various reactions.

7 ADC varies independently with values proportional to "fadc" and the proportionality factor also varies independently for each reaction. This allows one to determine the values of "fadc" or "fadc1" for various reactions, independently, using the options "NCOMP $=5$ " or "NCOMP $=6$ ", and then use the option "NCOMP $=7$ " to evaluate all reactions simultaneously.

NR $\quad=\quad 1$ to include $\sigma_{R}$ (the magnitude of the reaction cross-section) on the search in this data set ( 0 otherwise)

IPIT = Plotting parameter for the given reaction. If "IPLT" is zero the experimental data and the evaluated data will be recorded in the corresponding ".odf"-files

NT $=1$ to include $\sigma_{T}$ (the total cross-section) on the search in this data set ( 0 otherwise) 
NS $\quad=\quad$ Number of $\sigma(\theta)$ 's in this data set

NP $\quad=\quad$ Number of $P(\theta)$ 's in this data set

The parameters NR, NT, NS and NP satisfy,

$$
\mathrm{NR}+\mathrm{NT}+\mathrm{NS}+\mathrm{NP} \leq 100 .
$$

GENOA does not increase LMX if necessary. If LMX is too small to pass the criteria in the code, the user gets the printout "LMX TOO SMALL".

The integration of the radial equations is done, for each data set, up to a maximum radius, $R_{M}$, given by,

$$
R_{M}=R+8 a
$$

for which the nuclear potential is non-negligible. This criterium to define $R_{M}$ is similar to the one used in TNG's subroutine "chapso", in the file "chap.f", for the definition of the upper radial integration limit, "rmax", in the calculation of the transmission coefficients for the levels of the excited composite nucleus.

GENOA uses three sets of $R_{M}$ and $a$ and the largest among thein is used to cut off the radial integration. If $h$ designates the integration step length, then

$$
\frac{R_{M}}{h} \leq 147
$$

that is, the maximum number of mesh points is 150 .

- For maximum speed of the code the maximum number of partial waves. "LMX", should be set equal to the required number plus a few for safety. A good criteria for LMX required is [49]

$$
\mathrm{LMX}=k R_{M}-\eta
$$

A similar criterium is used by TNG in the definition of the maximum angular mommentum quantum number "is" in subroutine "chapso", in "chap.f".

In Eq.(B.2) $R_{M}$ is given by Eq.(B.1) and $\eta$ is the Coulomb parameter, which appears in the quantum description of the scattering of a charged particle by an electrostatic potential, given by,

$$
\eta=\frac{Z_{1} Z_{2} e^{2}}{(\hbar c)(v / c)}
$$

and the other parameters are, 
$\mathrm{Z} 1, \mathrm{Z} 2=$ number of electric charges present in the interacting particles

$e^{2}=$ square of the electric charge of the electron (1.439975 MeV frn )

$(\hbar c)=$ Planck's constant times the velocity of light $(197.32859 \mathrm{MeV} \mathrm{fm})$

$(\mathrm{v} / \mathrm{c})=$ relative velocity of the particles in units of "c"

Card 8: Title Card for each reaction. Format $12 \mathrm{A5}$.

Use the first 60 columns of this Card for title or comments

\section{B.3 SPECIFICATION OF DATA POINTS FOR EACH SET}

Card 9: $1^{\text {st }}$ set of Cards, for $\sigma(\theta)$ 's. Format (3F10.3), i.e., 1 Card per angle

$$
\left.\begin{array}{cl}
\theta= & \text { c.m. angle in degrees, } \\
\sigma(\theta)= & \text { c.m. x-section in mb/str (differential XS), } \\
\Delta \sigma(\theta)= & \text { error in x-section in per cent, } \pm 10 \% \text { error is } 10 .
\end{array}\right\} \text { NS Cards }
$$

Card 10: $2^{\text {nd }}$ set of Cards, for $P(\theta)$ 's. Format (3F10.3), i.e., 1 Card per angle

$$
\left.\begin{array}{rl}
\theta= & \text { c.m. angle in degrees, } \\
P(\theta)= & \text { polarization, } 20 \% \text { is } 20 . \\
\Delta P(\theta) & =\text { error in per cent of } P(\theta), \pm 4 \% \text { is } 4 .
\end{array}\right\} \text { NP Cards }
$$

Example: At 20 degrees the polarization measurement is $(0.30 \pm 0.04)$.

In this case, the user must write

$$
\theta=20 ., \quad P(\theta)=30 \text {. and } \quad \Delta P(\theta)=4 \text {. }
$$


Card 11: Reaction cross-section. If $N R=1$. Format free

$\sigma_{R}$ in $\mathrm{mb}$,

$\Delta \sigma_{R}$ in per cent of $\sigma_{R}$, i.e., $10 \%$ is 10 .

Card 12: Total cross-section. If NT $=1$. Format free

$\sigma_{T}$ in $\mathrm{mb}$,

$\Delta \sigma_{T}$ in per cent of $\sigma_{T}$

Card 13: Total compound elastic scattering cross section "CKP", in mb, and the "fadcp" parameter. Format free.

"CKP" is a parameter used in the definition of the Compound Elastic component of the total cross-section. "fadcp" is used in connection with the option "NCOMP $=7$ ", in Card 7 above. It is the independently variable proportionality factor which multiplies "fadc".

Card 14: "(AKP(L), L=1,9)". Format (9E10.4)

The components of "AKP" are the Lagrange coefficients to be used in the definition of the Compound Elastic part of the total cross-section. These coefficients are calculated by TNG to improve the evaluation of the differential cross-section by GENOA.

\section{B.4 COMMENTS OF FRANCIS G. PEREY ON THE OUTPUT OF GENOA}

We reproduce here some final comments of Ref. []․

"It is difficult to tell if a search has converged or not."

"Due to the internal workings of the code, if the parameters do not vary much, for successive central guesses, it does not mean that a minimum has been reached."

"In general, a local minimum has very likely been reached if", for all central parameters, "the absolute value of $\Delta X$, in percent of $X$ are srnall, say, less than $1 . "$

"The output which follows the OM parameters at each central guess is useful to find out how the search went." The user's sensibility to this information "increases with increasing experience and detailed knowledge of how the code works." 


\section{APPENDIX C - DESCRIPTION OF TEMPORARY FILES OF GENOA AND TNG}

We give here a description of the temporary files open by GENOA and TNG as a consequence of the segmentation procedure described in Sec.II.

"SAM11.DAT"

This file is opened in connection with the source file "wrt69.f" for transferring the data of the container array "a(n)"

"SAM14.DAT" This file is opened only once at the beginning of the GENOA-TNG procedure in the file "/gen/sgenoa.f" and is used in "/gen/buffeg.f" to store updated information on the parameter "ADC".

The parameter "ADC" was originally used by GENOA to evaluate the magnitude of the total compound-elastic cross-section, simultaneously with the search of Optical Model parameters, to obtain a minimum chi-square.

In the original definition, the energy-dependent magnitude of the compound elastic contribution was represented by the empirical relation

$$
\sigma_{C E}(E)=A /[1+\exp [(B-E) / C]]
$$

where each parameter, "A", "B" and "C", was associated to an independent component of the array "xcal".

This possibility corresponds to setting the pararneter "NCOMP" equal to "2" for the corresponding reaction. Presently GENOA uses six different options to define "ADC", corresponding to "NCOMP" equal 1,2, .., 6. The various options are defined in Card 7 of Appendix B.

"SAM16.DAT" This file is opened in the subroutines "wrt16x" and "wrt16" of the source file "/mas/mmas4.f" in connection with the master procedure of SAMMY.

"SAM29.DAT" This file is used in connection with subroutine "timer", in the file "/end/msamxx.f", to calculate the time spent by SAMMY in each segment 
"SAM30.DAT"

"SAM31.DAT"

"SAM46.DAT"

"SAM47.DAT"

"SAM49.DAT"
This file is opened in "sgenoa.f", "mmpw.f" and "rnmpw0.f" in connection with the MPW procedure (see Sec.IV.2.1.) to store the data of the arrays

"wwwwww" $=[\mathrm{W}]=$ the variation of the parameter covariance-matrix

and

"xxxxxx" $=[Y]=$ the approximate first order variation of the theoretical

parameters with respect to the experimental data.

This file is opened in the source file "/mas/mmas8.f" in connection with the master procedure of SAMMY.

This file is opened in "sgenoa.f" and "mmpw1.f" in connection with covariance matrix in MPW procedure, it stores:

The updated parameter covariance-matrix $[\mathrm{M}]=$ "vrprx(i)"

The inverse of updated parameter covariance-matrix $[\mathrm{M}]^{-1}=$ "vrprnv(i)"

The diference: (old-parameters) - (current-parameters) = "delpar(i)"

This file is opened in "sgenoa.f" and "mmpwl.f" in connection with covariance matrix in the MPW procedure.

It stores the initial parameter covariance-matrix $M$ "vrpr(i)" in subroutine "nrout0", to be read in "mmpw1.f".

Initially, "M" is assumed to be diagonal with non mull components given by the square of the originally defined values of the "variations" of the central-parameter array "xcal"

The values of "tsp" (the original "variations" of "xcal") are given as input in "fort.50" (unit-50).

This fle is opened in the source files "sgenoa.f", "mrnpw.f" and "rnmpw0.f" in connection with the evaluated central-parameters in MPW procedure.

The subroutine "newpar", in the source fle "mmpw1.f", solves the Bayes equations to update the central-parameters, using MPW procedure.

The updated and the old parameters are stored in the container array "a(n)" and are transferred to GENOA with the help of file "SAM49.DAT".

The new parameters are stored into the array "xcen", in subroutine "nrout0", to evaluate the convergence. 
"SAM69.DAT"

"STGCEN.DAT"

"STG10.DAT"

"fort.13"

"STGA18.DAT"

"STGA38.DAT"

"STGA51.DAT"

"STGA52.DAT"

"fort. $53 "$

"STGA54.DAT"
If the convergence has not been achieved the components of "xcen" are transferred back to "xcal" to begin a new numerical iteration.

This file is opened in connection with "wrt69.f" for transferring the data of the common blocks included in the file "TRANSF", except the information of the container array "a(n)".

This file is opened in "sgenoa.f" and "wrt150.f", stores the updated centralparameters, "xcen", to be used in the em redefinition of the input file of GENOA, "fort.50", if the input parameter "icrun" is bigger than one (see "icrun" in Card 1 of Appendix B. )

This file is opened in "sgenoa.f", "tngl.f" and "buffeg.f". It stores the the physical parameters of the Optical Potential, in subroutine "buffeg", to be read and used by the segment "samtn1" of TNG (source file "tng1.f")

This file is opened in "iogeno.f", "wrt150.f", "sgenoa.f" and "tngl.f" stores information to be used in the redefinition of the input file of GENOA, "fort.50", if the input parameter "icrun" is bigger than one, see Card 1 of Appendix B.

In particular, it stores the total compound elastic scattering cross section which is the parameter "CKP" in GENOA and "tcnst" in TNG.

TNG writes, in file "fort.62", a series of compound cross sections and Legendre coefficients which correspond to the fundamental and excited states of the residual nucleus in the final channel.

GENOA uses only the first set, corresponding to the compound elastic component. Auxiliary file not related to data transference among segments of SAMMY. Auxiliary file not related to data transference among segments of SAMMY. Auxiliary file not related to data transference among segments of SAMMY. Auxiliary file not related to data transference among segments of SAMMY. Auxiliary file not related to data transference among segments of SAMMY. Used in connection with the MPW procedure.

Auxiliary file not related to data transference among segments of SAMMY. Used as data file for generating plotting files. 
"STGA55.DAT"

"STGA56.DAT"

"STGA57.DAT"

"STGA58.DAT"

"STGA59.DAT"

"STGO61.DAT"

"STGO62.DAT"

"STGA71.DAT"

"STG72.DAT"

"STG8.DAT"
"STG95.DAT"

"STG96.DAT"
Auxiliary file not related to data transference among seginents of SAMMY. Keeps track of the sequence of input data of GENOA along the whole job.

This file is opened in "wrt150.f", to be used in the redefinition of the input file of GENOA, "fort.50", if the input parameter "icrun", in Card 1 of Appendix B, is bigger than one.

This file is opened in "tngl.f". It is an auxiliary file not related to data transference among segments of SAMMY.

This file is opened in "tng1.f". It is an auxiliary file not related to data transference among segments of SAMMY.

This file is opened in "tngl.f". It is an auxiliary file not related to data transference among segments of SAMMY.

Output file of TNG, is opened in "tng1.f", and is not related to data transference among segments of SAMMY.

Output file of TNG, is opened in "tng1.f", and is not related to data transference among segments of SAMMY.

This file is opened in "wrtl50.f". It is an "index" file, to be used in the redefinition of the input file of GENOA, "fort.50", if the input parameter "icrun", in Card 1 of Appendix B, is bigger than one.

This file is opened in connection with "wrt69.f", for transferring the data of the common block "common/factor/FACTIK". It is used to update the array "FACTIK", in connection with the evaluation of convergence in GENOA.

Auxiliary file not related to data transference arnong segments of SAMMY.

Auxiliary file not related to data transference arnong segments of SAMMY. It is used in connection with subroutine "run", in the file "msamxx.f", to store the pathnames of the next segment of SAMMY to be executed.

This file is opened by GENOA in subroutine "nrout0", in the source file "/git/sgenoa.f", to store the information of the following arrays, "FCEN", "FEXP", "WEIGHT", "sig", "xcomp", "EPS", "wwwwww", "xxxxxx" and "delpar"

to be transferred between two sequential calls of "nrouto". 
This file is opened in subroutine "idimen", in "/end/msamyy.f", in connection with the definition of the size of the various sub-arrays of the container "a(n)".

"STG99.DAT" This file is opened in subroutine "timer", in "/end/msamxx.f", in connection with the definition of the time of execution of the various segments of SAMMY. 


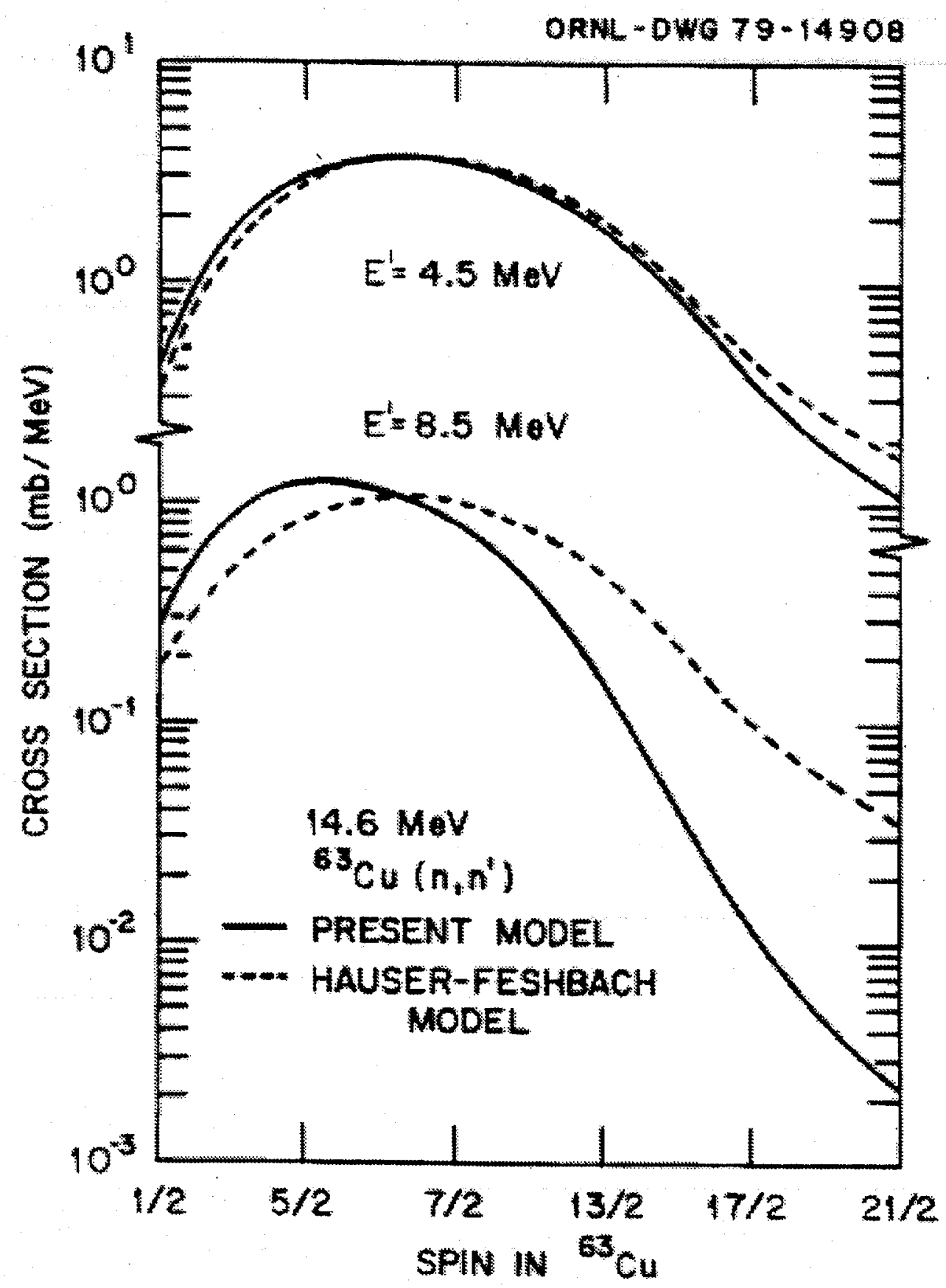

FIG. 1. Spin populations in ${ }^{63} \mathrm{Cu}$ following $14.6-\mathrm{MeV}\left(\mathrm{n}, \mathrm{n}^{\prime}\right)$ reaction for two outgoing neutron energies, $E=4.5 \mathrm{MeV}$ and $E=8.5 \mathrm{MeV}$. The solid curves are based on the model implemented in TNG, which includes precompound effects. The dashed curves are based on the Hauser-Feshbach method. 


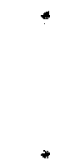


ORNL/TM-2000/252

\section{INTERNAL DISTRIBUTION}

1. Laboratory Records

2-3. Laboratory Records (OSTI)

4. H. Derrien

5-9. C. Y. Fu

10. N. M. Greene

11. K. Guber

12-16. F. B. Guimarães

17. D. T. Ingersoll

18. P. E. Koehler

19. M. A. Kuliasha
20. D. C. Larson

21. N. M. Larson

22. L. C. Leal

23. C. V. Parks

24. R. W. Roussin

25. R. 0. Sayer

26. M. S. Smith

27. T. Valentine

28. R. M. Westfall

29. RSICC

\section{EXTERNAL DISTRIBUTION}

30. H. Beer, Forschungszentrum Karlsruhe, IK, BAV 425, Postfach 3640, D-76021 Karlsruhe, Germany.

31. R. Block, Rensselaer Polytechnic Institute, Troy, NY 12180-3590.

32. O. Bouland, DER/SPRC/LEPH, Batiment 230, Centre dEtudes de CADARACHE, 13108 Saint Paul-lez-Durance, France.

33. D. Cabrilla, U.S. Department of Energy, NE-40, 19901 Germantown Road, Germantown, MD 20874-1290.

34. D. E. Carlson, Reactor and Plant System Branch, Division of System Research, Office of Nuclear Regulatory Research, U. S. Nuclear Regulatory Commission, MS T-10 G6, RM T-10, 17, Washington, DC 20555-0001.

35. M. B. Chadwick, Los Alamos National Laboratory, MS B243 T-16 Los Alamos, New Mexico 87545.

36. R. L. Dintaman, U.S. Department of Energy, DP-13, Washington, DC 20585.

37. C. Dunford, Bldg 197D, National Nuclear Data Center, Brookhaven National Laboratory, Upton, NY 11973.

38. J. R. Felty, U.S. Department of Energy, DP-311, Washington DC 20585.

39. P. Finck, Argonne National Laboratory, Reactor Analysis Division, Bldg 208, Argonne, IL 60439.

40. C. M. Frankle, NIS-6, MS J562, Los Alamos National Laboratory, Los Alamos, NM 87545.

41. F. Froehner, Kernforschungszentrum Karlsruhe, Institut f. Neutronenphysik und Reacktortechnik, Postfach 336 40, D-76021 Karlsruhe, Germany.

42. W. Furman, Frank Laboratory of Neutron Physics, JNR, Dubna, Russia.

43. S. Ganesan, Head, Nuclear Data Section, Indira Gandhi Centre for Atomic Research, Kalpakkam 603 102, Tamilnadu, India.

44. F. Gunsing, Centre D'Etudes De Saclay, F-Saclay - 91191 Gif-sur-Yvette Cedex, France.

45. G. M. Hale, T-2, MS B243, Los Alamos National Laboratory, Los Alamos, NM 87545.

46. A. Hasagawa, Nuclear Data Center, Japan Atomic Energy Research Institute, Tokai-mura, 
Naka-gun, lbaraki-ken 319-11, Japan.

47. R. N. Hwang, Argonme National Laboratory, Reactor Analysis Division, Bldg 208. Argonne, IL 60439

48. R. P. Jacqmin, DER/SPRC/LEPH, Batiment 230, Centre D'Etudes de CADARACHE, 13408 Saint Paul-lez-Durance, France.

49. N. Janeva, Bulgarian Academy of Sciences, 72, Boul, Tzarigradsko shosse, Sofia 1784, Bulgaria.

50. F. Kappeler, Forschungszentrum Karlsruhe, IK, BAV 425, Postfach 3640, D-76021 Karlsruhe, Germany.

51. L. Lambros, 08 E23, U.S. Nuclear Regulatory Commission, 11555 Rockville Pike, Rockville, MD 20852-2746.

52. R. Little, X-TM, MS B226, Los Alamos National Laboratory, Los Alamos, NM 87545.

53. C. Lubitz, Knolls Atomic Power Laboratory, P. 0. Box 1072, Schenectady, NY 12301.

54. R. E. MacFarlane, T-2, MS B243, Los Alamos National Laboratory, Los Alamos, NM 87545.

55. C. Mounier, CEN Saclay, DMT/SERMA/LENR, 91191 Gif Sur Yvette Cedex, France.

56. D. Muir, IAEA Nuclear Data Section, Wagramerstr. 5, P. 0. Box 100, A-1400 Wien, Austria.

57. C. Nordborg, OECDNEA, Le Seine St-Germain 12, Boulevard Iles, 92130 Issy-les-Moulineaux, France.

58. C. Raepsaet, CEN Saclay, DMT/SERMA/LEPP, 91191 Gif Sur Yvette Cedex, France.

59. M. Salvatores, DRN/P, Batiment 707, C. E. CADARACHE, 13108 Saint Paul-lez-Durance, France.

60. E. Sartori, OECDNEA, Le Seine St-Germain 12, Boulevard Iles, 92130 Issy-les-Moulineaux, France.

61. 0. A. Shcherbakov, Petersburg Nuclear Physics Institute, 188350 Gatchina, Leningrad District, Russia.

62. R. Shelley, Central Bureau for Nuclear Measurements, Steenweg op Retie, 2240 Geel, Belgium.

63. K. Shibata, Nuclear Data Center, Japan Atomic Energy Research Institute, Tokai-mura, Naka-gun, lbaraki-ken 319-11, Japan.

64. A. B. Smith, TD 362 D216, Argonne National Laboratory, Argonne, IL 60544.

65. D. L. Smith, TD-360-L106, Argonne National Laboratory, Argonne, IL 60544.

66. H. Takano, Nuclear Data Center, Japan Atomic Energy Research Institute, Tokai-mura, Naka-gun, lbaraki-ken 319-11, Japan.

67. C. Wagemans, Central Bureau for Nuclear Measurements, Steenweg op Retie, 2240 Geel, Belgium.

68. H. Weigmann, Central Bureau for Nuclear Measurements, Steenweg op Retie, 2240 Geel, Belgium.

69. C. Werner, Renssalaer Polytechnic Institute, Troy, NY 12180-3590.

70. R. White, Lawrence Livermore National Laboratory, P. 0. Box 808, Livermore, CA 94550.

71. M. Williams, Nuclear Science Center, Louisiana State University, Baton Rouge. LA 70803.

72. Phillip G. Young, Los Alamos National Laboratory, MS B243 T-16 Los Alamos, NM 87545. 